\title{
Revisión y análisis bibliométrico de la investigación sobre automedicación desde el año 2000
}

\author{
Érika Marcela Mora Gómez \\ Andrés M. Pérez-Acosta \\ Universidad del Rosario \\ Bogotá, Colombia
}

\author{
Recibido: 27 de junio del 2017 / Aceptado: 15 de enero del 2018 \\ doi: https://doi.org/10.26439/persona2018.n021.1990
}

La automedicación constituye una práctica transcultural, extendida a la población de todas las edades. Dicha práctica gira en torno a la más vaga concepción de autocuidado o preservación del bienestar biológico de la persona, de la prevención de la enfermedad $y$ de la promoción de la salud. Sin embargo, puede ser tan perjudicial como el hecho de no tratar las condiciones que representan una amenaza para la salud. Este hecho da lugar a que se planteen interrogantes en el ámbito cientifico. El presente artículo propone presentar el conjunto de variables más frecuentemente estudiadas en la literatura científica, desde el 2000 hasta la actualidad, sobre el comportamiento de automedicación. Se llega pues a dos tipos de conclusiones de esta revisión bibliométrica: reafirmar el establecimiento global del fenómeno de la automedicación y conocer la tendencia constante de variables ceñidas a este comportamiento, de alto riesgo para la persona que lo practica.

\section{automedicación / medicamento / prescripciones / bibliometría}

\section{Review and Bibliometric Analysis of Research on Self-Medication Since Year 2000}

Self-medication is a transcultural practice which includes populations of all ages. Said practice focus on the vaguest conception of people's self-care or biological well-being preservation, illness prevention, and health promotion. However, self-medication can be as harmful as not treating the conditions that threaten health. This situation results in raising questions in the scientific field. The present article aims at presenting the set of variables regarding self-medication that have been most frequently studied in scientific literature since year 2000 until now. Two different conclusions arise from this bibliometric analysis: to confirm the global establishment of self-medication phenomenon, and to find out the constant trend of variables linked to this high-risk behavior.

self-medication /medication / prescriptions/ bibliometrics 


\section{INTRODUCCIÓN}

Entre las generalidades del comportamiento humano, se encuentra la de guardar y promover su propio bienestar en diferentes ámbitos, para la preservación de la vida. La automedicación constituye una práctica transcultural que afecta a la población de todas las edades y gira en torno a conceptos tales como autocuidado o resguardo del bienestar de la persona, prevención de la enfermedad y promoción de la salud.

Si bien se destaca como una práctica con intenciones de conservar y mejorar la salud, lo cierto es que puede provocar perjuicios o consecuencias serias a las personas que la ejercen. Usualmente, puede convertirse en la primera opción de una persona para menguar síntomas o condiciones de salud desfavorables. Es decir, pueden existir cogniciones del ser humano que le permitan empoderarse y sentirse autosuficiente para emprender un plan de acción concreto, que constituya un autotratamiento. $\mathrm{Y}$ es que si no llega a funcionar dicho tratamiento, la preocupación por la salud se incrementa, lo cual lleva a recurrir a otros métodos de autotratamiento o a acudir donde un profesional de la salud.

En el presente trabajo se abarcarán, en primera instancia, los conceptos de percepción del estado de salud, conductas de autocuidado y promoción y prevención en salud; los que darán pie para que el fenómeno de la automedicación se plantee alrededor de estos. Luego, se realiza un desglose de todos aquellos factores y elementos que contribuyen a la promoción de la automedicación; finalmente, se plantean los riesgos de esta práctica tan común que, a su vez, justifican los objetivos y el planteamiento de este estudio.

\section{Autocuidado, promoción y prevención de la salud}

A lo largo de la historia, el ser humano ha habitado en ambientes diversos que demandan el ejercicio de habilidades para sobrevivir. Pero no de cualquier manera; por el contrario, de la mejor forma posible: por ejemplo, gozando de una salud estable. Entonces, es válido destacar el concepto de cuidado. En la concepción de Heidegger, la persona, ante la angustia o fenómenos que le causen preocupación por sí misma, acude al cuidado, que es finalmente su propio ser. Es decir, se concibe como un interés puramente existencial y no intelectual, y un constructo inherente al ser humano (Botero, 2007).

Conociendo que el cuidado es inevitable y un accionar que es natural e inherente a todo individuo, el autocuidado puede introducirse como la tendencia del ser a realizar comportamientos o conductas que promuevan el bienestar propio y que eviten los estímulos que puedan generar perjuicio o inestabilidad de dicho bienestar ideal. Sería, entonces, el hecho de agregar al concepto de cuidado una decisión voluntaria de la persona por emprender acciones que la conduzcan a un estado óptimo de salud.

El autocuidado, definido por Escobar, Franco y Duque (2011), se establece en tres supuestos indispensables: 1) ser un proceso de carácter voluntario por y para 
la persona misma; 2) ser una filosofía de vida ligada a experiencias previas y basada en redes de apoyo, y 3) estar apoyado en un nivel de conocimiento básico que promueva intercambios y relaciones interindividuales. Estos supuestos giran en torno a esfuerzos del ser humano; pues la responsabilidad de dicho constructo recae en este. $\mathrm{Y}$ es que la misma palabra genera dicha responsabilidad (auto).

El autocuidado puede ser definido como una serie de "prácticas cotidianas y las decisiones sobre ellas, que realiza una persona, familia o grupo para cuidar de su salud" (Tobón, 2003). Según Tobón (2003), esta práctica se acopla al ser en forma de destrezas aprendidas a lo largo de la vida, y tienen por objetivo fortalecer o restablecer la salud y prevenir la enfermedad. Adicionalmente, aclara que este conjunto de prácticas responde a la capacidad de supervivencia y a conductas habituales de la cultura en la que se encuentra la persona que ejecuta el autocuidado.

Otra de las definiciones que se da al autocuidado es la que proporciona Orem, quien afirma que es aquella "conducta aprendida por el individuo, dirigida hacia sí mismo y el entorno para regular los factores que afectan su desarrollo en beneficio de la vida, salud y bienestar" (citado por Vega y González, 2007). En resumen, el autocuidado trata de un constructores inherente y vinculado a la capacidad de aprendizaje del ser humano, que se va desenvolviendo en una serie de acciones ligadas a la necesidad individual de conservar o mejorar la salud en todas sus expresiones, y que varía de acuerdo a especificaciones culturales del individuo que lo practica.

Por su parte, Silva y colaboradores (2009) contribuyen a la definición de autocuidado al afirmar que no se trata de un constructo innato al ser, como lo había manifestado inicialmente Heidegger, sino de una actividad adquirida a través de procesos de aprendizaje individuales, orientada hacia un logro específico; en cuyo caso se hablaría de actividades dirigidas a promover el bienestar.

Conociendo algunas nociones alrededor del concepto de autocuidado, que bien lo mencionen como un elemento innato al ser o como un aprendizaje individual de este, es importante distinguir dos palabras que se usan cotidianamente con relación al fenómeno de la automedicación y que pesan lo suficiente como para dar paso a este comportamiento en el ser humano: la promoción de la salud y la prevención de la enfermedad.

Por una parte, el concepto de promoción de la salud se liga a la palabra promover, que significa "dar impulso a, fomentar, originar, generar" (Ferreira, 1986; citado por Czeresnia, 1999). Leavell y Clark (1976) definen promoción de la salud como una serie de medidas que no tienen por meta dirigirse a una enfermedad o desorden en específico, sino que, por el contrario, son útiles para aumentar la salud y bienestar generales (Czeresnia, 1999). También, se concibe como la creación de condiciones que constituyan el sustento del bienestar, mediante el fortalecimiento de acciones de verificación, 
acrecentamiento y mejora de la salud propia (De Roux, 1994).

Por otro lado, la prevención de la enfermedad se relaciona con la palabra prevenir, que significa, en términos de Ferreira (1986): "preparar, llegar antes de, disponer de manera que evite, impedir que se realice". Entonces, el concepto de prevención de la enfermedad estaría definido como toda acción anticipada que se basa en el conocimiento de la historia natural a fin de hacer improbable que la enfermedad se expanda de manera posterior (Czeresnia, 1999). Por ello, es necesario, como bien lo menciona De Roux (1993), el fundamento de la persona sobre el conocimiento de la etiología y racionalidad de aquellos comportamientos de riesgo, con la finalidad que el plan de acción que este mismo elabore se sustente en un conjunto de factores entrelazados que justifiquen, al mismo tiempo, el carácter perjudicial para la salud.

Al diferenciar estos dos términos, se entiende que ambos se enfocan en una meta que gira en torno a la salud. Sin embargo, la manera en que persiguen esta meta es lo que los distingue. Como bien menciona Uribe (1999), la diferencia entre promoción y prevención "guía las opciones que cada persona asume frente al cuidado". Ambos conceptos tienen por agente un individuo que genera un plan de acción, sea para promover o prevenir, salud o enfermedad, respectivamente. Se parte, entonces, de que la promoción de la salud y prevención de la enfermedad se vinculan directamente con el autocuidado, en el modo en que ambos se centran en acciones autogeneradas, valga la redundancia, por un individuo que busca fortalecer su bienestar en la medida de sus posibilidades, o reducir todo riesgo de enfermedad que pueda generar daño a la salud que tiene.

\section{El fenómeno de la automedicación}

Si hay que definir la palabra automedicación, se diría, desde el concepto más básico, que es la medicación ejercida por y para sí mismo. Sin embargo, es importante, en primera instancia, atender a lo que la etimología nos dice sobre esta palabra compuesta. Desde el punto de vista etimológico, la palabra automedicación puede fraccionarse en dos segmentos: auto y medicación. Según la Real Academia Española (RAE), el prefijo auto significa, desde la etimología griega, "propio" o "por uno mismo". Y el segundo segmento, "medicación", se deriva del latín medicatǐo, entendido como la administración de medicamentos con un fin terapéutico. Es válido unir estos dos segmentos para definir, de manera preliminar, la automedicación como la acción de una persona de administrarse a sí misma un medicamento con fines terapéuticos.

Sylvie Fainzang, de modo literal expresa, en su artículo acerca de la automedicación, que esta se concibe como el uso de medicamentos basado en la propia decisión de un sujeto en específico (2013). Por su parte, Bergmann (2003) afirma que un medicamento de automedicación es todo aquel específicamente 
adecuado para su uso sin la prescripción o recomendación de un médico.

El resultado de la inferencia realizada y las primeras observaciones a lo que es automedicación, respectivamente, generan una serie de interrogantes: ¿Dicho medicamento ha sido prescrito por un profesional de la salud o ha sido el propio individuo quien ha decidido qué, cómo y cuándo administrárselo en su propio organismo? ¿La acción de medicación ha estado motivada por el propio individuo u otras situaciones han catalizado o fomentado este proceso? ¿Qué tipos de medicamentos entran a ser parte de esta definición, solo aquellos que se adquieren sin prescripción médica? Después de todo, abordar el término automedicación no es tan sencillo como parece. A continuación, se presentará una serie de definiciones que permitirán, finalmente, aterrizar un poco más el concepto central de la investigación.

Lecomte considera a la automedicación como un proceso más complejo, en el que un individuo realiza un autodiagnóstico y despliega una serie de acciones con el objetivo de perfeccionar un tratamiento sin recibir ningún tipo de asistencia médica. Y lo amplía al sostener que se trata del poder de adquisición de una sustancia sin la prescripción debida (1999, Citado por Fainzang, 2013). La definición que proporciona Granda (2008) enfatiza a la automedicación como una forma de autoatención, en la cual la ingesta de medicamentos se origina por iniciativa propia del paciente, a partir de una experiencia previa o sin ella.
Asimismo, Orueta, Gómez-Calcerrada y Sánchez (2008) definen este fenómeno como una situación en la que los pacientes se basan en decisiones propias para adquirir y utilizar medicamentos, sin que el médico participe de la prescripción o la supervisión del tratamiento.

Es interesante notar que en estas definiciones el sujeto principal en la acción de automedicación se identifica con el rol de paciente, no es simplemente una persona. Esto puede dar a entender que es posible que exista alguna condición por la cual la referida persona está atravesando por unos síntomas o características de una enfermedad, o bien ha desarrollado una condición de enfermedad. Aun así, estas definiciones continúan siendo amplias y generales, por lo cual es válido pensar en este fenómeno de una manera más particular y ajustar algunos de los cabos que pudiesen estar sueltos.

Por ejemplo, Loyola Filho, LimaCosta y Uchôa (2004) describen la automedicación en términos del tipo de tratamiento terapéutico que se usa y de la clase de actividad que se desarrolla, a partir del deseo de conservar o mejorar el estado de salud. Entonces, respecto al tipo de tratamiento terapéutico identificado, la automedicación puede concebirse como una práctica que se ejecuta alrededor del consumo tanto de medicamentos de la industria farmacéutica, como de remedios caseros, que tal vez están ligados a constructos netamente culturales. Respecto al tipo de actividad generada dentro del fenómeno de la automedicación, se señalan los siguientes: adquirir 
medicamentos sin la debida prescripción, presentar recetas médicas antiguas a fin de obtener medicamentos, usar medicamentos que han sobrado de tratamientos terapéuticos previos y aún se conservan en casa, o incluso no adherirse a las recomendaciones médicas, modificando, interrumpiendo o prolongando la dosis recetada originalmente.

Fainzang (2013), después de mencionar ciertos aspectos aislados de lo que es automedicación, resalta que al final puede concebirse como: "el uso de un medicamento por iniciativa propia, sin consultar a un médico por el problema en cuestión, bien sea que el medicamento sea propiedad del individuo o bien que este último procure tenerlo (en una farmacia o de otro individuo". (p. 494)

De otro lado, Grahame-Smith alude a la existencia de dos tipos de automedicación que conducen al afinamiento de la descripción de esta actividad. El primero se basa en situaciones en las que un individuo acude a una farmacia y adquiere un medicamento de venta libre o sin receta. El segundo tipo se identifica con la manipulación de la profesión médica (1975). En cuanto a esta propuesta, especialmente en lo que se refiere al segundo tipo de automedicación, es importante identificar que no solo aquí se puede ver al sujeto que hace las veces de paciente, sino al que, siendo profesional de la salud o en formación, se encuentra en rol de paciente y toma ventaja de sus conocimientos para ejercer la automedicación. Es importante tener en cuenta esta definición, ya que posteriormente se verá como un factor de influencia en el ejercicio de esta práctica.

Haciendo un análisis de las definiciones expuestas en los aportes de los autores mencionados, se pueden identificar los siguientes aspectos comunes:

Primero, la existencia de un sujeto; independientemente de que ejerza un rol de paciente actual o no, que genera prácticas para modificar su estado de salud o conservarlo. En todo caso, para promover su salud y prevenir la enfermedad, generando bienestar propio.

Segundo, el espacio dado para la acción de toma de decisiones; si bien el sujeto se encuentra en $x$-situación, que genera un incentivo y motivación lo suficientemente significativos como para que active cogniciones y planes de acción hacia la meta: el bienestar propio, lo más completo posible.

Tercero, un plan de acción guiado a la adquisición de $x$-medicamento(s) mediante terceros para emprender un autotratamiento. Este plan de acción varía dependiendo de la circunstancia del individuo. Si el individuo cuenta con una atención médica anterior respecto a una misma condición de enfermedad o similar, el plan puede basarse en repetir el tratamiento recomendado previamente. Por otro lado, si el paciente acude a un profesional de la salud, quien le extiende una receta, con la dosis y el tratamiento que debe seguir, el individuo recae en automedicación si asume un comportamiento autónomo que implica modificar las indicaciones que le dieron. Y si el individuo no acude a los servicios médicos 
prestados por un sistema de salud, su plan de acción podría estar basado en conductas que desafían la profesión médica y se desvinculan de todo tipo de recomendaciones por parte de los profesionales de la salud.

Cuarto, el ejercicio del plan de acción mencionado por cuenta propia; una vez adquiridos los tratamientos terapéuticos expresados, sea en medicamentos farmacéuticos de venta libre o no, o en remedios caseros.

Quinto y último, la existencia de riesgos potenciales en algunos de los casos en los que la automedicación cruza la delgada línea entre el autocuidado y el abuso de información terapéutica de promoción de la salud y prevención de la enfermedad.

\section{Factores que promueven la práctica de automedicación}

La automedicación, basada en las decisiones propias del individuo acerca de su condición o estado de salud, se fundamenta también en elementos o factores que justifican su desarrollo. Constantemente, el individuo toma decisiones que, en ocasiones, no surgen de manera unidireccional; por el contrario, las decisiones varían de acuerdo a un conjunto de factores que determinan el curso de las acciones que emprenderá.

Respecto a la práctica de automedicación, existen numerosos factores relacionados con el origen y curso de este fenómeno, que están estrechamente vinculados con la cercanía del individuo a lo que él concebiría como sistema de salud. Es decir, el individuo puede aproximarse a dicho constructo de sistema para la atención y tratamiento de condiciones desfavorables mediante distintas vías: un profesional de la salud directamente, publicidad, experiencias propias o cercanas a él que dictan el curso de un tratamiento, o simplemente constructos de tipo cultural que pasan de generación en generación, por ejemplo, en el voz $a$ voz. A continuación, se realiza una revisión de algunos factores.

Género. En ocasiones, surge la pregunta sobre la influencia del género en diversas tomas de decisiones; si el género masculino es de una forma u otra, o si el género femenino es de esta o aquella forma, tenderá a realizar acciones de una manera determinada y distinta del género opuesto. Es bien sabido,que, a lo largo de muchos años, se ha generado una guerra de sexos alrededor de múltiples temas. Por supuesto, la automedicación no está exenta de esta contienda.

En algunas investigaciones se ha expuesto al género femenino como el más longevo en comparación al masculino, ya que un mayor porcentaje de mujeres reporta frecuentemente su enfermedad, usando más los servicios de cuidado de la salud. Antes de explorar la vulnerabilidad de un género u otro a recaer en comportamientos de automedicación, sería crucial atender a aspectos que conciernen al cuidado de la salud en hombres y mujeres.

Verbrugge (1985), basándose en estadísticas recolectadas en los Estados Unidos, afirma que las mujeres de entre 17 y 44 años acuden el doble número de 
veces a visitas médicas y estadías en la clínica en comparación con los hombres. Hacia la edad de 45 años, no son tan frecuentes las visitas; sin embargo, siguen representando un porcentaje mayor. Asimismo, en el uso de medicamentos de prescripción y de venta libre, las mujeres superan a los hombres en un $50 \%$. Verbrugge concluye que las diferencias de género en cuanto al cuidado de la salud se fundamentan en el hecho que las mujeres se enferman de manera más frecuente, pero con menos riesgo que los hombres; por lo cual su asistencia a servicios de salud, en un promedio anual, es mayor que la del género masculino.

Desde este punto de vista, podrían inferirse argumentos como:

Las mujeres, por acudir más seguido al médico son más propensas a automedicarse; es decir, como se exponen más al conocimiento de los tratamientos, terminan almacenándolos en su mente. En cambio, los hombres serían menos vulnerables a recaer en dicha actividad, porque solo acudirían a la asistencia médica en ocasiones necesarias y de fuerza mayor.

Las mujeres se automedican menos, pues recurren a asistencia médica en vez de confiar en comportamientos de automedicación; los hombres se automedican más porque el hecho de acudir al médico por sintomatología más riesgosa los lleva a conductas de automedicación, cuando presentan sintomatología de bajo riesgo.

Cualquiera de las premisas anteriores es tan apresurada que es importante revisar el comportamiento de automedicación para los dos géneros.
Payne, Neutel, Cho y Desmeules (2004) al usar, en su estudio, los datos de tres periodos anuales del National Population Health Survey (NPHS) -1994-1995, 1996-1997 y 1998-1999para hombres y mujeres de 20 años; centraron su atención en la pregunta: Durante los últimos dos días, ¿cuántos medicamentos, sean prescritos o de venta libre, tomó?. El estudio se cruzó con variables tales como matrimonio y familia, educación, empleo e ingresos. En todas las variables, el resultado de la revisión sugiere que una mayor proporción de mujeres reporta el uso de medicamentos.

Hasta el momento, se observa que el género femenino es un agente potencial para desarrollar más conductas de automedicación que el género masculino, sin caer en estereotipos. Sin embargo, autores como Klemenc-Ketis, Hladnik y Kersnik (2011) revelan datos alrededor de las diferencias de género en la automedicación que contrarrestan los de estudios anteriores. Estos autores realizaron un estudio transversal anónimo, empleando un cuestionario online, en la Universidad de Liubliana (la más grande de Eslovenia). El cuestionario usado recabó información demográfica, y de actitudes y prácticas generadas en torno al comportamiento de automedicación. Los resultados revelan que el género no evidenció influencia alguna en la incidencia del fenómeno. Aun así, la población femenina que participó (991 mujeres equivalentes al 76,6 \% de la población objeto) fue más cautelosa en el uso de algunos medicamentos y respecto la automedicación como tal, pues constituye una actividad que no es 
completamente segura, especialmente por la presencia de efectos secundarios.

Respecto a esta contienda de géneros, incluso se puede encontrar información empírica sobre la calidad de los medicamentos consumidos por representantes de cada género. Por ejemplo, Sanfélix, Palop, Pereiró y Martínez realizaron un estudio de tipo transversal analítico, contando con población masculina y femenina de 14 años en adelante que acudiera a los centros de salud de la comunidad valenciana, en seis meses. Al clasificar los medicamentos como Vie (valor intrínseco elevado) y Viene (valor intrínseco No Elevado), resultó que las mujeres de entre 45 y 64 años consumen más medicamentos de Vine; es decir, de baja calidad, con relación a aquellos que usan los hombres. Esto es, un porcentaje de 1,8\% contra $0,4 \%$, respectivamente (2002).

En síntesis, el debate sobre automedicación, diferenciando géneros, nos da por resultado un carácter neutral, al equiparar a los géneros en cuanto a su vulnerabilidad a esta práctica común. Ello porque no se pueden realizar inferencias partiendo únicamente del hecho de ser hombres o mujeres, más bien, es posible realizar una revisión de factores que no son tan genéricos y que, en cambio, pueden tener influencia tanto en hombres, como en mujeres, a la hora de recurrir en comportamientos de automedicación.

Profesionales de la salud. Este factor puede dividirse en dos grandes ramos: tener una influencia directa de un profesional de la salud para adquirir un medicamento con la finalidad de establecer un autotratamiento, o bien el de ser un profesional de la salud y tomar ventaja de dicho título para ejercer automedicación. La primera opción derivada de este factor se sostiene en la situación en la cual una persona que asume el rol de paciente en un momento específico acude a un profesional de la salud, no necesariamente un médico, aunque es posible, a fin de obtener información de productos, técnicas o cualquier tipo de tratamiento para aliviar síntomas o molestias establecidas a lo largo de un periodo de tiempo determinado. Dentro de esta opción, puede que esta persona conozca a alguien cercano que sea profesional de la salud, en formación o no, quien aparenta tener los conocimientos suficientes como para confiar en los consejos que da con relación al mejoramiento o estabilidad de la salud de aquella persona.

Destaca, de modo común, la influencia de farmaceutas o auxiliares de farmacia en la adquisición de medicamentos para atender síntomas o simplemente realizar tratamientos ligados a la promoción de la salud y prevención de la enfermedad. Una de las investigaciones al respecto fue realizada en Santa Catarina, Brasil. Los investigadores tomaron una lista de todas las farmacias registradas en esta ciudad y las dividieron por distritos. Una vez hecho esto, enviaron a dos actrices a cada farmacia que simulaban tener una hermana enferma en casa, con temperaturas de entre $38.5^{\circ} \mathrm{C}$ y $40{ }^{\circ} \mathrm{C}$, y dolor de cabeza. Las actrices pedían a quien las atendiera en la farmacia que les proporcionaran un antibiótico para su hermana enferma. En caso de que surtiera 
efecto, le preguntaban a esta persona que las atendía si era farmacéutico. En el $74 \%$ de las 107 farmacias visitadas, las actrices obtuvieron el antibiótico sin tener prescripción médica. En cuanto a quienes atendían la farmacia, 70 resultaron ser farmacéuticos y 37, empleados de ventas. De los que eran farmacéuticos, el $84 \%$ proporcionaría antibióticos a un cliente, si lo considerara necesario (Espíndola, et al., 2005).

Barbero-González y colaboradores (2006) realizaron un estudio observacional transversal en farmacias de España, teniendo como objetivo evaluar la solicitud y dispensación de medicamentos que requieren de receta médica, sin tener la debida prescripción. Se contó con la participación de 139 farmacias de origen rural y urbano; se encontraron 6205 pedidos de medicamentos de prescripción, de los cuales se otorgó el 97 \% (6020 medicamentos). Es importante también destacar los motivos por los cuales los farmacéuticos dispensaron los medicamentos y sorprendentemente destacan los hechos que eran medicamentos recomendados por un médico y que los clientes ofrecieran traer la receta médica unos días después.

Otro estudio, realizado en Estambul con 73 farmacéuticos, recabó información por medio de observación y entrevistas a lo largo de las diferentes estaciones (verano, invierno, otoño y primavera) acerca de la distribución de medicamentos que requieren de prescripción o no. Se encontró que el $41 \%$ del total de medicamentos dispensados correspondía a aquellos no prescritos. Adicional a ello, los farmacéuticos afirmaron que no solo los clientes demandan medicamentos sin prescripción, sino que también requieren información sobre los medicamentos sin necesidad de adquirirlos (Gül et a., 2007).

Ahora bien, la segunda opción identificada dentro de este factor se refiere a individuos que estarían cumpliendo dos roles, los cuales se afectan mutua y simultáneamente por voluntad propia. Es decir, por una parte, estarían asumiendo el rol de pacientes, con unos signos y síntomas propiamente dichos $\mathrm{y}$, por otra, estarían cumpliendo el rol de profesionales de la salud; es decir, médicos de sí mismos. Siendo médicos, sus conocimientos en salud, cuidado de la salud, fármacos, tipos de medicamentos y sus efectos, sugeriría que, en caso de presentar complicaciones en su propia salud, no considerarían necesario consultar otro profesional de la salud. Hem y colaboradores realizaron un estudio a lo largo de nueve años con estudiantes de medicina graduados entre 1993 y 1994, a quienes les aplicaron cuestionarios un año, cuatro años y diez años después de haberse graduado. Los resultados revelaron que la mayoría de médicos se había autoprescrito medicamentos: el $90 \%$ en la primera etapa del estudio, el $86 \%$ en la segunda y el $84 \%$ en la tercera. Solo el $10 \%$, en las tres fases del cuestionario, respondió que había acudido donde otro profesional para obtener la medicación necesaria (2005).

Por otro lado, Schneider, Gallacchi, Goehring, Künzi y Bovier llevaron a 
cabo un estudio en el que seleccionaron, de manera aleatoria, a 1784 médicos de atención primaria en Suiza. El estudio se basó en un cuestionario mediante el cual se recabó información acerca de la asistencia a profesionales de la salud o servicios médicos, en el último año, para atender alguna problemática de salud propia, y el uso de algún medicamento (analgésicos, opiáceos, benzodiacepinas, antidepresivos, entre otros) durante la última semana. Se concluyó que solo el $21 \%$ de los participantes contaba con un médico al que recurría de manera regular. Adicional a ello, el $53 \%$ consultó con algún profesional de la salud y alrededor del $65 \%$ (1152 participantes) reportó el uso de medicamentos farmacéuticos en la última semana; de este último grupo, el comportamiento de automedicación se observó en el $90 \%$ de los casos (2007). Los estudios mencionados anteriormente se refieren a personas que son médicos; no obstante, no están exentos los representantes de las farmacias ni aquellos individuos que se están formando como profesionales de la salud.

Tong y Lien (1995) afirman que la automedicación tiene mayor prevalencia en aquellos profesionales con fácil acceso a medicamentos de prescripción, tales como farmacéuticos y médicos. En su estudio tuvieron como propósito determinar hasta qué punto los representantes de las farmacias hacen mal uso de las muestras de medicamentos. Durante los meses de abril y junio, se pidió a 27 representantes farmacéuticos que contestaran una encuesta sobre el uso de las muestras que les dispensaban. Dentro de los resultados, se identificó que 48,1 \% de los participantes (13 farmacéuticos) dieron sus muestras a otras personas (no médicos), como a sus amigos, cónyuges, padres o hermanos. El resto de participantes $(14,8 \%)$ afirmó haberse automedicado con dichas muestras.

Por otro lado, otros de los agentes que tienen conocimiento acerca del cuidado de la salud, además de información sobre tratamientos y efectos de ciertas sustancias y medicamentos, son aquellos profesionales de la salud en formación. Ansam Sawalha entrevistó a 1581 estudiantes, entre los cuales algunos pertenecían a la facultad de medicina y otros no. Se encontró que los estudiantes de facultades diferentes a la de medicina tenían mayores niveles de automedicación (98,6 \%). Es importante reconocer que debido a sus pocos conocimientos médicos, estos hacen uso de diversos medicamentos para aliviar un síntoma específico, mientras que los estudiantes de facultades de medicina, con 96,6\% de niveles de automedicación, tienen mayor probabilidad de tratarse a sí mismos con el medicamento apropiado desde el primer momento en que presentan uno o varios síntomas (2008).

Acceso al sistema de salud. Sería, en cierto modo, fácil reconocer que, debido a los altos costos del sistema de salud, muchos de los pacientes de bajos recursos económicos encuentran difícil acceder a estos; por lo cual, se trataría de una población aún más vulnerable a recaer en comportamientos de automedicación. 
En la ciudad de Riyadh, capital de Arabia Saudí, se propuso el estudio de comportamientos de automedicación, para el que se contó con la participación de pacientes que acudieron a centros de atención primaria de la salud. Las respuestas de 500 encuestas contestadas, sus resultados revelaron que 177 pacientes —el 35,4 \% de esta muestra- desarrollaron conductas de automedicación en las últimas dos semanas. Dichas conductas se reflejaron en la adquisición de medicamentos de venta libre en farmacias privadas, donde el rol del farmacéutico volvió a jugar un papel importante; o simplemente se debió a medicamentos de tratamientos pasados que les sobraron y aún estaban en posesión de los pacientes, quienes recordaban las indicaciones que les dieron. El 70,1 \% de los pacientes que contestaron la encuesta afirmó que uno de los motivos para automedicarse era que las facilidades del sistema de salud no estuvieran disponibles ante la necesidad de estas, y el 42,9 \% reportó que el costo de las consultas médicas con un profesional de la salud le impedía asistir y tener que asumir la autoridad en el asunto, automedicándose (Alghanim, 2011).

En comunidades o regiones que carecen de suficientes recursos económicos, la mayoría de enfermedades son tratadas mediante automedicación, ya que dicho estatus socioeconómico hace casi imposible la adquisición de medicamentos modernos de alto costo; e incluso la presencia de doctores en áreas rurales es casi nula; lo cual da lugar a diversas dificultades acerca del acceso al sistema de salud. La consecuencia de ello es la automedicación, no siempre con medicamentos intervenidos por la industria farmacéutica, sino con opciones más económicas; como productos herbarios y plantas, que juegan un rol importante dentro de este fenómeno (Shankar, Partha y Shenoy, 2002).

Es importante reconocer que no solo elementos socioeconómicos intervienen en el acceso al sistema de salud. Independientemente de si los pacientes cuentan o no con los recursos económicos para gozar de atención a su salud, todo paciente posee un sistema psicológico que le permite, por medio de sus percepciones, realizar un feedback con relación a la satisfacción que recibe al ser atendido por profesionales de la salud. Grigoryan, et al. afirman que dentro de los factores determinantes para que algunos pacientes de países con bajos recursos se automediquen con antibióticos se encuentra, además del costo de la consulta médica, la baja satisfacción ante la atención de los médicos (2008).

Cuidadores. Este factor aplica especialmente para individuos que pueden practicar de manera indirecta la automedicación. Es decir, niños y adolescentes que, a pesar de tener capacidad de decisión, aún se encuentra en desarrollo y no está lo suficientemente estructurada. Por ello, las decisiones sobre la adquisición de un medicamento o tratamiento en especial corren por cuenta de quienes cumplen el rol de cuidadores. Los cuidadores pueden ser los mismos padres, o bien personas a quienes fue delegada 
la función de hacerse cargo del niño o adolescente.

Es posible que los niños sean quienes estén bajo más influencia de la toma de decisiones de sus cuidadores, respecto a comportamientos de automedicación. Por su parte, los adolescentes podrían estar o no bajo dicha influencia; cuando no lo están, son ellos mismos quienes emprenden acciones hacia la automedicación (Du y Knopf, 2009). Esto podría explicarse por el hecho de que, en edades más avanzadas, es posible que se hayan experimentado o presenciado situaciones en las que la automedicación ha sido efectiva o exitosa, las cuales generan en el paciente adolescente la confianza de que cuantas veces suceda, tantas veces la automedicación funcionará.

En el estudio realizado por Pereira, Bucaretchi, Stephan y Cordeiro (2007) se incluye la automedicación en población infantil y adolescente. Este estudio consistía en entrevistar a los padres o tutores de los participantes que eran menores de 18 años, quienes habían consumido algún medicamento en los últimos 15 días. De 772 padres o tutores entrevistados, en 437 casos se afirmó haber consumido medicamentos en los últimos 15 días. Adicional a ello, el análisis reveló que la práctica de automedicación es más frecuente en grupos de edades mayores (entre 7 y 18 años), independientemente de su género y condiciones socioeconómicas.

Por otro lado, la revisión realizada por Payne y colaboradores se observa desde la perspectiva del cuidador. En este estudio, respecto a la variable matrimonio $y$ familia, los padres, que tienen niveles de educación altos e hijos pequeños en casa, presentan una menor proporción en el uso de medicamentos, mientras que los padres con niveles de educación bajos han incrementado la utilización de medicamentos en casa (2004).

Observando las premisas resultantes de los estudios anteriores, podría pensarse que, en edades de 0 a 6 años la automedicación se encuentra en menor magnitud, debido a que posiblemente los padres o tutores piensan en que sería irresponsable de su parte atender las quejas de los niños sin consultar con un profesional de la salud. Igualmente, podría explicarse en el hecho de que en edades mayores los pacientes son más estructurados a la hora de expresar qué les está causando molestia; por lo cual los padres o tutores podrían tomar decisiones unidireccionales que se inclinen hacia la disminución o extinción de la molestia en el paciente.

Es importante reconocer que la actuación de los cuidadores ante la presencia de una enfermedad en el niño o adolescente es muy significativa, pues son ellos quienes toman decisiones acerca de la manera de proceder ante dicha condición desfavorable. El hecho de emprender acciones antes de consultar a un profesional de la salud puede empeorar la enfermedad e incluso generar riesgos más alarmantes y perjudiciales para el paciente.

Soriano et al. (2009) exponen el caso de un grupo pediátrico de 87 niños con infección respiratoria aguda, de los cuales 47 fueron automedicados por sus cuidadores. La automedicación se presentó con 
el uso de antihistamínicos, vasoconstrictores o antigripales y remedios caseros, como tés de pitiona y gordolobo. Se menciona que este comportamiento ocurrió durante un tiempo mayor a 72 horas, en el que hubiera podido darse un manejo desde el sistema de salud propiamente dicho, de la mano de un profesional. El $17 \%$ de los pacientes refirieron empeoramiento de su condición clínica, y el 6,4 \% reportó mejoría. Entre las consecuencias desfavorables en este caso preciso, estuvo la intervención con mecanismos de defensa naturales, lo que causó una posible intoxicación, que generó signos y síntomas adversos, adicionales a la condición inicial del paciente.

Otra de las particularidades de los cuidadores se muestra cuando ejercen el rol de la maternidad. Una madre puede estar automedicando a su hijo, en cuanto decida emprender acciones de este estilo desde su condición de embarazo. Dentro de esta investigación se asumirá el estado de embarazo como aquel en el que una madre lleva un ser vivo dentro de su vientre. Sharma, Kapoor y Verma confirman que alrededor del $8 \%$ de embarazadas se ven en la necesidad de pasar por tratamientos basados en medicinas debido a enfermedades de tipo crónico o complicaciones propias de su estado. Por ello, el embarazo es un estado en el cual los tratamientos que incluyen el uso de fármacos debe ser sumamente cuidadoso por su posible efecto teratogénico, que por supuesto concierne a la criatura en desarrollo (2006).

En el estudio realizado por Miní et al. (2012) en Perú se propuso identificar las causas potenciales por las que las gestantes se automedican. Con este propósito claro, se aplicó un cuestionario a 400 gestantes mayores de 19 años respecto a conductas de automedicación durante dicho estado. Los resultados de este estudio mostraron que algunas de las razones por las cuales las gestantes se automedican son: recomendación de amigos, familia, personal administrativo de salud o profesionales de salud no médicos; por falta de tiempo para acudir al médico o simplemente el acceso al servicio, y porque a pesar de que este tiene los recursos, se percibe lento y no disponible.

Asimismo, las gestantes que habían practicado previamente la automedicación fueron más propensas a desarrollar esta actividad durante su embarazo. Es importante mencionar que los medicamentos de uso más común en estado de gestación fueron aquellos de venta libre. Adicional a ello, estos autores identificaron que las gestantes presentaron tres posibles factores de riesgo para la automedicación: 1) desempleo; 2) subempleo, y 3) estar en el tercer trimestre del embarazo. Estos autores infieren que un nivel de educación alto y estar casada son circunstancias que posiblemente blindan a algunas gestantes para practicar la automedicación.

Publicidad. Desde el punto de vista histórico, Díaz-Caycedo, Payán-Madriñan y Pérez-Acosta (2014) mencionan la relevancia del capitalismo como causa altamente influyente en la prevención y curación de enfermedades, en la medida en que se genera, a través de estas actividades, una lógica de mercado que irónicamente 
desemboca en el hecho de que la sociedad justifique la automedicación por la confiabilidad que la publicidad genera.

Como bien mencionan Ras Vidal y Moya Ortiz (2005), la automedicación incluye variables inherentes a la población, como la cultura, creencias, costumbres, las que se perciben afectadas por los mecanismos de comercialización de las industrias farmacéuticas (Citados por Díaz-Caycedo, Payán-Madriñan, y Pérez-Acosta; 2014). Ello muestra el poder de la publicidad, que transforma la realidad de un producto en lo más agradable y acorde posible para una cultura específica (porque el mismo producto farmacéutico puede ser comercializado de distintas maneras, variando de una sociedad a otra), consiguiendo que quienes asumimos en un momento determinado el rol de consumidores compremos ideas que terminan convirtiéndose en la adquisición y uso de un producto de manera independiente.

La publicidad, grosso modo, es un mecanismo que usa un productor hacia un consumidor, cuyo propósito es lograr que dicho consumidor adquiera su producto. En el caso de los medicamentos, la publicidad realiza una influencia significativa desde la etiqueta, las propagandas mediante radio o televisión, la información del producto en internet, o simplemente los consejos del propio profesional de la salud que actuaría como un promotor del producto, voluntaria o involuntariamente.

Un estudio relacionado con automedicación pediátrica se enfoca en las malinterpretaciones que se presentan cuando los cuidadores o padres de niños menores de dos años de edad les suministran medicamentos de venta libre, con el objetivo de aliviar síntomas de resfriado y tos. Lokker y colaboradores (2014) contaron con la ayuda de 182 cuidadores, de los cuales el $87 \%$ cumplía el rol de madre; ellos contestaron un cuestionario acerca del uso de cuatro medicamentos para el resfriado y la tos, que en la etiqueta afirmaban ser para niños menores de dos años. Dentro de los resultados, se observó que más del $50 \%$ de estos cuidadores suministraría alguno de dichos productos a su hijo o niño a cargo, en caso de presentar síntomas de resfriado. Uno de los catalizadores para tomar esta decisión fue la etiqueta del medicamento que contenía la palabra infante (infant); las imágenes de elementos relacionados con la población infantil, por ejemplo, niños, osos de felpa o goteros, así como instrucciones sobre las dosis apropiadas. Si bien este estudio comprende la toma de decisiones por parte de un cuidador sobre acciones hacia la salud de un infante, también le concierne a efectos de publicidad que serán revisados posteriormente.

Con relación a esta problemática, María González de Cossío sugiere que en los productos farmacéuticos hay una gran responsabilidad para la automedicación. Entonces, para que existan comportamientos de automedicación lo más responsables posibles, es necesario que la publicidad del medicamento tenga en cuenta elementos informativos y aspectos de cuidado alrededor del medicamento y su contexto, de los usuarios 
del producto, del lenguaje que se usa para llegar al entendimiento del consumidor o usuario del producto, del uso de espacios en las etiquetas, entre otros (2008).

En Estados Unidos y en Nueva Zelanda se permite la publicidad de medicamentos de prescripción dirigida a los pacientes. Si bien esto halla su explicación en la gran inversión monetaria que se realiza en la industria farmacéutica, la publicidad de estos productos ofrece una importante cantidad y calidad de información que Mintzes y colaboradores decidieron estudiar (2006). Mediante una serie de encuestas en California y Vancouver, se evaluó si los pacientes que participaron hicieron pedidos correspondientes a nuevas recetas médicas o de productos promovidos directamente al consumidor mediante publicidad. De 1431 participantes, el $12 \%$ admitió haber requerido una nueva prescripción. $Y$ de este porcentaje, el $42 \%$ pidió exactamente productos promovidos por medio de publicidad.

Como en todo debate, la existencia de polos opuestos es imprescindible. En el caso de publicidad directa al consumidor, algunos afirman que tiene un plus, pues sirve como un recurso educativo para los usuarios directos o indirectos del producto, y otros sostienen que entre sus aspectos negativos está el contribuir a la medicalización de enfermedades de bajo riesgo, que incluso pueden ser síntomas triviales, lo que lleva a que se usen de manera incorrecta los productos farmacéuticos (Dieringer et al., 2011).
La bivalencia del asunto de la publicidad genera conflictos que se arraigan al cuidado de la salud y se comportan como un arma de doble filo. Los medicamentos, remedios o productos farmacéuticos son creados a fin de ejercer un efecto positivo en el organismo de un individuo o contrarrestar elementos negativos en este, pero su mal uso también es perjudicial. La absoluta ignorancia acerca del producto es tan perjudicial como la ignorancia de creer que el producto se está usando de la manera correcta.

Botiquines caseros. Habitualmente, en casa se cuenta con un botiquín o un lugar en donde se guardan y almacenan medicamentos por un tiempo indefinido. Espacio que, por lo general, se relaciona con tratamientos pasados o con productos que sugieren su uso frecuente por síntomas que se presentan una y otra vez, y parecen ser lo suficientemente triviales como para no acudir a un profesional de la salud. Dichos botiquines pueden ser perjudiciales en la medida en que se convierten en el primer recurso al que la persona recurre para atender y cuidar su salud.

Es conocido que todo medicamento requiere del debido cuidado para permanecer en óptimas condiciones y actuar de manera adecuada en el organismo de quien, con justa causa, se beneficia de él. Por eso, es importante conocer las fechas de vencimiento y las condiciones físicas en las que se debe tener el producto para no alterarlo. Sin embargo, este no es siempre el caso de los medicamentos que están guardados en botiquines caseros. 
Leen De Bolle et al. realizó un estudio sobre los botiquines caseros en el que estudiantes de quinto año de Farmacéutica debían entrevistar a quienes acudían ciertas farmacias comunitarias y conseguir una visita en las residencias de dichos clientes. Esto, usando un inventario que recababa información sobre los medicamentos guardados, el lugar de reserva y las unidades expiradas, y el propósito con el que se guardaban los medicamentos. En caso de ser automedicación, la frecuencia de uso y las dosis, entre otros. Se observó que de 288 clientes que visitaron la farmacia, el 92,4 \% guardaba los medicamentos en un lugar específico, seco y a temperatura fresca. Sin embargo, de los medicamentos que necesitaban ser refrigerados, solo el 14,6\% los tenía en condiciones adecuadas. Además, se encuentra que el $56 \%$ de participantes usaba los medicamentos guardados con propósitos de autotratamiento por propio criterio (2014).

Adicional a lo anterior, la presencia de menores en casa representa un peligro aún mayor, pues el fácil acceso al botiquín puede producir una intoxicación en los más pequeños. Como bien mencionaron Tourinho, Bucaretchi, Stephan y Cordeiro (2008) en su estudio, los lugares más usados para hacer reserva de medicamentos fueron habitaciones propias, cocina y baños, en $47,5 \%$, $28,9 \%$, y $14,6 \%$, respectivamente.

\section{Riesgos de la automedicación}

A lo largo del documento se ha venido explorando el trasfondo de constructos $\mathrm{y}$ factores que promueven el origen y desarrollo de comportamientos de automedicación en algunos individuos. Se ha dicho que la automedicación puede estar cargada de una intención o varias; de carácter positivo, lo cual, desde esta perspectiva lineal, se percibe como una actividad benéfica. Sin embargo, no todos los casos de automedicación son favorables para el individuo que la practica.

Muchas veces, el autotratamiento puede funcionar y generar en la persona un sentimiento de satisfacción y autosuficiencia, que descarta el conocimiento científico de aquellos profesionales de la salud que aportan al bienestar de sus pacientes. Como bien mencionan Sharma, Kapoor y Verma (2006), los medicamentos juegan un rol muy importante en la mejora de la salud y en la promoción del bienestar del individuo, de hecho, estos generalmente han sido creados con este propósito. Por lo cual, conseguir dichos ideales de salud y bienestar, significa que el medicamento debe ser seguro, eficaz y debe ser usado racionalmente.

Todo individuo que cumpla el rol de paciente en algún momento y que además se automedica está en riesgo de presentar perjuicios en su salud. Es importante observar a qué tipo de consecuencias se atiene un ser humano cuando intenta, por voluntad e iniciativa propia, atender a su salud mediante la automedicación.

El entendimiento de que existen, a grandes rasgos, dos grupos importantes de medicamentos guía esta discusión. Por un lado, se encuentran los medicamentos de venta libre (en inglés Over The 
Counter, OTC) y, por otro lado, están los medicamentos de prescripción únicamente (en inglés Prescription-Only Medication POM). Los medicamentos de venta libre son productos farmacéuticos para el alivio de síntomas de bajo riesgo, que el consumidor adquiere sin exponerse a grandes peligros, sin exonerarlos de ser perjudiciales, por ejemplo, en su consumo en exceso. Por su parte, los medicamentos de prescripción son un conglomerado de familias de medicamentos que requieren la autorización y supervisión médica debido al alto nivel de cuidado que se debe tener al consumirlos en un período de tiempo determinado.

Existen cuatro razones por las cuales la Unión Europea guía las prácticas del Reino Unido para el manejo de los medicamentos de prescripción (POM): 1) Existe un peligro, bien sea directo o indirecto, para la salud en el instante en el que el medicamento está siendo usado sin supervisión de un médico profesional de la salud, porque el uso incorrecto en dosis $\mathrm{y}$ efectos secundarios puede ser manejado por dicho profesional y puede salirse de control para el paciente; 2) los medicamentos pertenecientes a este grupo son erróneamente usados de manera común, por lo cual representan peligros inminentes para la salud y bienestar del paciente; 3) los efectos o acciones adversas del medicamento, después de haber sido ingerido, requieren de una rigurosa investigación por los profesionales de la salud, tanto antes como durante su consumo, y 4) el medicamento está siendo administrado de manera parenteral, lo que implica reacciones en el organismo que deben ser supervisadas (Bond $\mathrm{y}$ Hannaford, 2003).

Es erróneo pensar que solo los medicamentos de prescripción representan riesgos para la salud de un individuo; incluso los remedios más simples relacionados con hierbas naturales representan riesgos. Bond y Hannaford resaltan que una hierba medicinal llamada kafa kafa se ha visto particularmente relacionada con casos de hepatoxicidad alrededor del mundo. Tanto el tipo de medicamento, como su uso y manejo, se relacionan con los tipos de riesgos que se presentan. Estos autores también resaltan los peligros existentes con medicamentos de venta libre, ya que al ser libres de prescripción tiende a haber la creencia de que no son perjudiciales, por lo que el paciente tiende a incrementar las dosis y la frecuencia con las que toma este medicamento. Esto genera efectos adversos que, en algunos casos, no son reportados a ningún profesional de la salud (2003). Con respecto a los medicamentos de venta libre, Hughes, McElnay y Fleming (2001) sostienen que estos, por lo general, son recomendados para uso a corto plazo, pues su uso prolongado puede estar maquillando síntomas de una enfermedad mucho más grave y seria.

Previamente, se ha tratado el tema de aquellos profesionales que realizan prescripciones de medicamentos para sí mismos, hecho que sería concebido como un tipo de automedicación. Y es que en este tipo de población los riesgos que se señalan están ligados al abuso de medicamentos de prescripción, lo que lleva a la persona a presentar adicciones severas. 
Se ha propuesto, por lo menos en Victoria, Australia, medidas de tipo regulador y restrictivo que proporcionen un orden hacia aquellos profesionales que usan la autoprescripción (Rosvold y Tyssen, 2005).

Un riesgo importante para las personas que se automedican se fundamenta en la relación que establecen con el médico que las atiende. Independientemente de si la relación es buena o mala, algunos pacientes no mencionan las prácticas comunes que llevan de consumo de medicamentos o productos de la medicina alternativa; los cuales, combinados con medicinas prescritas, pueden agravar el problema de salud. Algunos pacientes pueden preferir no contarle a su médico por diversas creencias alrededor de esta figura profesional, o simplemente para manejar ellos mismos la conjugación de dos tratamientos. Es casi como si pensaran que el hecho de establecer dos o más tratamientos diferentes contribuirá a una mejor y más rápida curación. Hughes, McElnay y Fleming afirman que la combinación de ciertos medicamentos de venta libre con otros medicamentos puede generar sangrados excesivos, hipoglicemia, miopatía, crisis hipertensivas, entre otros (2001).

En la revista Gaceta Universitaria, en agosto de 1999, se publicó, en la sección de salud, "Los peligros de la automedicación”; allí, la Unidad de Protección Civil destacó seis aspectos negativos de este fenómeno:

1. El inadecuado uso de medicamentos puede originar intoxicaciones indeseables debido al desconocimiento de la prescripción en la especificidad de dosis, horario, duración, restricciones y otros.

2. La ingesta de dos o más medicamentos, simultánea e irresponsablemente, puede ocasionar interacciones de tipo inhibitorio o potencializador, y generar efectos colaterales (hemorragia, somnolencia, entre otros).

3. Retraso y dificultad en el diagnóstico de alguna enfermedad, debido a la modificación de signos y síntomas iniciales.

4. Presencia de reacciones alérgicas en la toma inicial o tras varias veces de la ingesta del medicamento.

5. Intoxicación, lo cual origina frecuentemente náuseas, vómitos, gastritis, visión borrosa, insomnio, daño hepático, etcétera.

6. Dependencia química o adicción, sobre todo en inhibidores del sistema nervioso central. Las consecuencias más graves oscilan entre una sobredosis que cause irritabilidad y somnolencia, hasta un paro cardiorrespiratorio.

Ahora bien, es usual el discurso sobre cierto grupo de medicamentos que genera cambios y afecta de manera negativa al organismo debido al mal uso, a su uso innecesario o a su uso prolongado. Baos (2000) destaca dos de los principales tipos de medicamento que se relacionan con riesgos potenciales para la salud de un ser humano: los analgésicos y los antibióticos. 
Por una parte, los analgésicos son, a nivel general, medicamentos relacionados con el alivio del dolor, desde dolor de cabeza hasta dolores musculares. El uso habitual de estos medicamentos, en su versión simple, es "nefritis intersticial", y se atribuye al consumo de estos medicamentos el $11 \%$ de casos de insuficiencia renal, lo que representaría 110 nuevos casos por cada 1.000.000 (Laporte, 1997; citado por Baos, 2000). En casos de cefalea, es muy común el uso de analgésicos, que en su empleo frecuente puede generar un ciclo repetitivo y a corto plazo de cefalea crónica diaria. Esto, a su vez, genera un ciclo perverso de abuso de los analgésicos, e incluso de elevar sus dosis (Baos, 2000).

Respecto a los antibióticos, existe una noción de que son para todo tipo de infecciones. Si se conoce la acción de los antibióticos, grosso modo, se entiende que estos combaten a los microorganismos bacterianos que están dentro de un ser humano. El consumo de antibióticos de manera continua, o bien el tratamiento de infecciones que no precisan de su consumo, pueden generar daños en la flora intestinal e incluso resistencia de las bacterias a los antibióticos que ya no serán efectivos. Vicente Baos menciona la gran prevalencia en España de una resistencia del $40 \%$ del streptococcus pneumoniae a la penicilina, así como la del escherichia coli y pseudomonas aeruginosa, en niveles preocupantemente altos, a antimicrobianos, como cefalosporinas (2000).

Teniendo conocimiento de estos aspectos perjudiciales, consecuencia de la automedicación, es momento de generar interrogantes que guíen estos comportamientos: ¿hasta qué punto la automedicación es perjudicial? ¿Qué tipo de medidas se deben tomar (educativas o legales) alrededor de la automedicación para que sea manejada justamente? ¿Qué otros factores están influyendo en que la automedicación, a pesar de representar de manera tangible (o experiencial) un perjuicio o varios para el organismo, continúe prevaleciendo?

\section{Pregunta de investigación}

¿Cuáles son las variables más frecuentes estudiadas en la literatura empírica sobre el comportamiento de automedicación?

\section{Objetivo general}

Identificar las variables más frecuentemente estudiadas en la literatura científica sobre el comportamiento de automedicación.

\section{Método}

\section{Muestra}

El presente trabajo constituye una investigación bibliométrica, elaborada a partir del análisis de 107 artículos de tipo empírico sobre el comportamiento de automedicación desde el 2000 hasta la actualidad (2014). Dichos artículos se encuentran publicados en revistas indexadas en PUBMED, principal base de datos especializada en ciencias de la salud.

La muestra y recurso principal de este artículo investigativo, una vez 
seleccionada, fue analizada individualmente. Ello permitió extraer, de cada una de las fuentes bibliográficas de tipo empírico, dos tipos de unidades de análisis que son significativas para la realización de observaciones alrededor del fenómeno de automedicación. Dichas unidades de análisis serán expuestas a continuación.

\section{Unidades de análisis}

Las unidades de análisis que se tendrán en cuenta son de dos tipos: bibliográficas y variables, estudiadas en cada uno de los artículos empíricos que componen la muestra.

En cuanto a las unidades de análisis bibliográficas, se incluye año de publicación, países en los que se ha realizado investigación empírica en automedicación, revistas en las que fueron publicados los artículos y autores de los artículos incluidos en la muestra. Por su parte, las variables estudiadas en la muestra de artículos empíricos abarcan edad, cuadros médicos, medicamentos, ocupación y estados especiales.

\section{Aspectos éticos}

De acuerdo a la legislación internacional de la APA, el presente trabajo está cuidadosa y especialmente cimentado en los principios éticos $\mathrm{C}$ y $\mathrm{E}$, integridad y respeto por los derechos y dignidad de las personas respectivamente, y en el código de conducta para los psicólogos (Ethical Principles of Psychologists and Code Of Conduct, 2002).

El principio $\mathrm{C}$, integridad, asegura que el psicólogo vele por presentar en la práctica de la profesión la precisión, honestidad y verdad en todo conocimiento científico, como el presentado en el actual trabajo.

El principio E, respeto por los derechos y dignidad de las personas, afirma que el psicólogo se compromete a guardar la dignidad y el valor de todo ser humano respecto a, por ejemplo, la privacidad, confidencialidad y autodeterminación.

Estos principios, aplicados al presente trabajo, se revelan de manera específica en los estándares 8.10 y 8.11 del manual de principios éticos psicológicos y normas de conducta de la APA. Dichos estándares exigen evitar el plagio a toda costa, mediante la debida citación de todos y cada uno de los aportes investigativos de los autores referenciados, como es el caso del presente trabajo.

En lo que respecta a la legislación colombiana, que reglamenta el ejercicio de la profesión de Psicología, el presente trabajo se fundamenta en los principios generales expresados en la Ley 1090 de 2006, especialmente el primero y el tercero.

El principio general número uno clama por la responsabilidad del psicólogo en todas sus actividades, aceptando las consecuencias de sus actos en el ejercicio de la profesión. Por su parte, el tercer principio general abarca los estándares morales y legales que permiten que el psicólogo se proclame como un ciudadano que respeta y es respetado por otros en toda actividad relacionada o no con su profesión (Ministerio de Protección Social, República de Colombia, 2006). 
Estos principios, aplicados al presente trabajo, adquieren sentido cuando se realizan los debidos procesos de citación de referencias usadas, para reafirmar el respeto a la propiedad intelectual de un colega o no, en lo que a la investigación respecta.

\section{Resultados}

Para todas y cada una de las unidades de análisis que genera la muestra estudiada, la extracción de los resultados se realiza mediante la identificación individual de cada dato por variable. Seguido este paso, se hace un conteo de cuantas veces aparece cada dato en la muestra de 107 artículos empíricos.

\section{Resultados bibliográficos}

Año. En lo que respecta al año de publicación, la presente revisión bibliométrica propone una exploración, en la base de datos PUBMED, de artículos que constituyan una investigación empírica alrededor del fenómeno de la automedicación, que hayan sido publicados desde el 2000 hasta la actualidad -2014(Figura 1). Observando esta figura, se pueden apreciar los años estudiados (eje horizontal) y la cantidad de artículos de la muestra publicados en cada año (eje vertical). Aunque entre el 2000 y 2004 se puede observar un bajo indicador de publicación con tendencia al decremento, para el intervalo 2004-2005, se identifica un incremento importante de seis artículos. Es a partir de allí y hasta el año 2009 que se observa un crecimiento progresivo en la publicación de artículos. Sin embargo, para el periodo 2009-2010 se percibe un descenso de diez artículos con rápida recuperación hasta el $2012(2012=12$ artículos), pero nuevamente caen las cifras para los años 2013 y 2014 con una estabilidad de cinco artículos.

Distribución global. Se identificaron, por su parte, 57 países en los cuales se ha realizado propiamente investigación sobre el fenómeno de la automedicación. Dichos países se encuentran distribuidos alrededor del mundo, con al menos un país por continente. En la Figura 2 se puede contemplar la distribución global. El continente que, como se observa, cuenta con concentración de investigación empírica en el tema central de este trabajo es Europa, seguido por América, especialmente en el Sur. Y el continente con menos concentración de investigación para la muestra estudiada es Australia.

Revistas cientificas. Respecto a las revistas en las que fueron publicados los artículos, ante la observación directa a cada uno de estos (Anexo A) y el conteo respectivo del número de artículos que aparecen adscritos a una de las 74 revistas identificadas en la muestra, se encuentra que la revista Pharmacy World \& Science (ahora conocida con el nombre de International Journal of Clinical Pharmacy) puntea cinco (5) veces en la muestra, seguida por la revista Family Practice con cuatro (4) artículos adscritos a esta. Las revistas Atención primaria, Annals of Pharmacotherapy, Journal of Antimicrobial Chemotherapy, Jornal de Pediatría (Río J.), BMC Public Health y 
BMC Health Services Research puntean, cada una, con tres (3) artículos adscritos a ellas. El resto de revistas, exactamente 66 , puntean con dos o un artículo adscritos a ellas.

Autores. Por último, se cuenta el número de veces en que aparece cada autor incorporado en la muestra de artículos incluidos en el presente estudio. (Anexo B) Se identificaron 437 autores diferentes dentro de la muestra. De estos, Grigoryan, L.; Bugerhorf, J. G. M.; Degener, J. E.; Stalsby, C.; Monnet, D. L.; Scicluna, E. A.; Birkin, J.; HaaijerRuskamp, F. M.; Bucaretchi, F.; Stephan, C.; Cordeiro, R.; De Bolle, L., Mehuys, E., Remon, J-P. , y Van Bortel, L. aparecen cada uno tres (3) veces en la muestra estudiada, y son los que encabezan la lista. El resto de autores (422) se observan una o dos veces en la muestra. Es importante mencionar que algunos de los autores con más apariciones colaboran en un mismo artículo.

\section{Resultados por variables estudiadas en la muestra}

Edades. Dentro de la variable edad, se encuentran cuatro grandes grupos con la finalidad de que no se traslapen entre sí, como se puede apreciar en la Tabla 1: 1) población exclusivamente infantil; 2) población exclusivamente adolescente; 3) población infantil y adolescente en un mismo estudio, y 4) población adulta, entendida como personas de 18 años en adelante. La amplitud y extensión de este último grupo de edades se explica por el hecho de que una proporción significativa de estudios se concentraba en la población general. Al respecto, se puede decir que el estudio en población adulta (o población general) es líder con 95 veces en la muestra, lo que representa el $88.7 \%$. Los estudios en población exclusivamente infantil y exclusivamente adolescente puntúan tres (3) veces, y representa cada uno el $2.8 \%$ en la muestra. Por último, los estudios de

\section{Tabla 1}

Distribución por grupo de edades de la población estudiada en las referencias empíricas sobre automedicación

\begin{tabular}{lc}
\hline Grupo de edades & $N^{\circ}$ de veces que aparece en la muestra \\
\hline Población infantil & 3 \\
Población adolescente & 3 \\
Población infantil y adolescente & 6 \\
Población adulta (18 años en adelante) & 95 \\
\hline Total & 107 \\
\hline
\end{tabular}


población infantil y adolescente puntúan seis (6) veces en la muestra, lo cual representa el $5.6 \%$.

Cuadros médicos. A diferencia de la variable edad, es inevitable que la de cuadros médicos no se traslape entre sí. Algunos cuadros parecieran solo ser síntomas; sin embargo, se le denominarán cuadros médicos para su fácil clasificación dentro del estudio. Una vez contado el número de veces que aparece en la muestra, se identifican los resfriados como la cabeza de la lista (Tabla 2) con trece (13) veces, seguido de cefaleas con doce (12) veces. Estas, a su vez, seguidas por problemas del tracto gastrointestinal y tos, con once (11) veces en la muestra cada uno. La hipertensión, diabetes y fiebre continúan la lista, cada una puntea diez (10) veces en la muestra de 107 artículos. Con ocho (8) veces en la muestra, aparecen tanto las enfermedades cardiovasculares como los síntomas menstruales, seguidos por trastornos mentales, dolor de garganta y síntomas musculoesqueléticos, con siete (7) veces que aparecen en la lista. Entre los cuadros que llaman la atención en la lista se encuentran: infecciones de transmisión sexual, úlceras, psoriasis, hipotiroidismo, cáncer o tumores malignos, entre otros.

Tabla 2

Tipo de cuadro médico y número de veces que aparece en la muestra

\begin{tabular}{lc}
\hline Cuadro médico & $N^{\circ}$ de veces que aparece en la muestra \\
\hline Resfriado & 13 \\
Cefalea & 12 \\
Problemas del tracto gastrointestinal & 11 \\
Tos & 11 \\
Hipertensión & 10 \\
Diabetes & 10 \\
Fiebre & 10 \\
Enfermedades cardiovasculares & 8 \\
Síntomas menstruales & 8 \\
Trastornos mentales & 7 \\
Dolor de garganta & 7 \\
Síntomas musculoesqueléticos & 6 \\
Dolor abdominal & 5 \\
Enfermedades respiratorias & 5 \\
Problemas relacionados con la vista & 5 \\
Cólico abdominal & 5 \\
\hline & \\
\hline & \\
(continúa)
\end{tabular}


(continuación)

\begin{tabular}{|c|c|}
\hline Migraña & \\
\hline Enfermedades del riñón o tracto uri & \\
\hline Gripe & 4 \\
\hline Osteoporosis & 4 \\
\hline Asma & 4 \\
\hline Problemas ginecológicos & 3 \\
\hline Heridas / Lesiones & 3 \\
\hline Cáncer o tumor maligno & 3 \\
\hline Náuseas & 3 \\
\hline Problemas dermatológicos & 2 \\
\hline Rinosinusitis no complicada & 2 \\
\hline Reumatismo o artrosis & 2 \\
\hline Artritis & 2 \\
\hline Infecciones de transmisión sexual & 2 \\
\hline Bronquitis & 2 \\
\hline Enfermedades de las articulaciones & 2 \\
\hline Dolor dental & 2 \\
\hline Fatiga & 2 \\
\hline Hipotiroidismo & 2 \\
\hline Dolor de oído & 2 \\
\hline Úlcera & 2 \\
\hline Candidosis Vaginal & 1 \\
\hline Malaria & 1 \\
\hline Acné & 1 \\
\hline Congestión nasal & 1 \\
\hline Amigdalitis aguda & 1 \\
\hline Faringitis & 1 \\
\hline Derrame cerebral & 1 \\
\hline Neoplasias & 1 \\
\hline
\end{tabular}


Medicamentos. En cuanto a los medicamentos usados por la población estudiada dentro de los artículos de la muestra, se puede observar cuatro tipos de medicamentos que encabezan el listado (Anexo C): 1) antibióticos, con 38 veces; 2) analgésicos, con 23 veces; 3) vitaminas, con dieciocho (18 veces), y 4) Antiinflamatorios no esteroideos, con 17 veces en la lista. Estos cuatro medicamentos están seguidos, con doce (12) veces en la lista por medicamentos para el tratamiento de enfermedades gastrointestinales y medicamentos herbarios, a su vez seguidos por tranquilizantes (11 veces en la muestra) y antialérgicos (10 veces en la muestra). Luego, aparecen nueve (9) veces cada uno en la muestra: los medicamentos para el tratamiento de enfermedades respiratorias, antiparásitos y medicamentos para tratar los resfriados. A estos los siguen los anticonceptivos y medicamentos dermatológicos, que aparecen ocho (8) veces en la lista. Entre otras medicinas que se pueden observar, están los medicamentos homeopáticos, laxantes, insulina, antimaláricos, antidepresivos, medicinas alopáticas, ayurveda y umami, entre otros. Es válido mencionar que los medicamentos también se traslapan entre sí en su conteo.

Ocupación. Dentro de las variables que reporta la muestra, está la ocupación de las personas que desarrollan la automedicación como actividad individual personal o promueven la actividad, que es fuente de provisión de los medicamentos. En la Tabla 3 se encuentra el listado de ocupaciones, en el cual los líderes de este fenómeno son los estudiantes de educación superior (11 veces en la muestra), seguidos por estudiantes de educación secundaria, médicos de atención primaria en salud y farmacéuticos, cada uno aparece tres (3) veces en la muestra.

\section{Tabla 3}

Ocupación de la población estudiada en las referencias empíricas sobre automedicación y número de veces que aparece en la muestra

\begin{tabular}{lcl}
\hline Ocupación & $N^{\circ}$ de veces que aparece en la muestra \\
\hline Estudiantes de educación superior & 11 \\
Estudiantes de educación secundaria & 3 \\
Médicos de atención primaria en salud & 3 \\
Farmacéutico & 3 \\
Staff médico & 2 \\
Estudiantes de educación primaria & 1 \\
Auxiliares de farmacia & 1 \\
Comerciantes (mujeres) & 1 \\
\hline
\end{tabular}


(continuación)

Trabajadores no profesionales de la salud 1

Soldadores 1

Empleados de bodega 1

Proveedores de salud 1

Trabajadoras sexuales 1

Si anteriormente se mencionaba que los estudiantes de educación superior encabezaban la lista de ocupaciones, es importante destacar a cuál área de pregrado está adscrita la población estudiada en la muestra de artículos empíricos, recabados para la realización de esta investigación.
Los pregrados que predominan son: medicina, enfermería y odontología. Dentro de la lista también se encuentran pregrados del área de la salud, como farmacología, nutrición, ciencias biológicas y psicología. (tabla 4).

\section{Tabla 4}

Tipo de pregrado de la población adscrita a estudios superiores

\begin{tabular}{lc}
\hline Tipo de pregrado (Pregrado en...) & $\mathrm{N}^{\circ}$ de veces que aparece en la muestra \\
\hline Medicina & 2 \\
Enfermería & 2 \\
Odontología & 1 \\
Farmacología & 1 \\
Veterinaria & 1 \\
Nutrición & 1 \\
Ciencias Biológicas & 1 \\
Educación Física & 1 \\
Psicología & 1 \\
Artes visuales & 1 \\
Ingeniería de Alimentos & 1 \\
Geografía & 1 \\
Derecho & 1 \\
Biología marina & 1 \\
\hline
\end{tabular}

Estados especiales. Esta variable se extrae como resultado de la existencia de casos que no se encuentran ligados a ninguna de las variables desarrolladas previamente. Se destacan tres estados especiales: 1) paternidad o cuidadores; 2) embarazo, y 3) maternidad, específicamente el caso único en la muestra de las 
madres nodrizas. En la Tabla 5 se puede apreciar que el estado o caso de paternidad o presencia de cuidadores aparece cinco (5) veces en la muestra de artículos empíricos recabados, seguido de tres (3) artículos que se centran en la etapa de embarazo de la mujer, ligada al fenómeno de la automedicación.

\section{Tabla 5}

Estados especiales asociados a la práctica de automedicación evidenciadas en la muestra estudiada

\begin{tabular}{lc}
\hline Estado & $N^{\circ}$ de veces que aparece en la muestra \\
\hline Paternidad o cuidadores & 5 \\
Embarazo & 3 \\
Maternidad (madres nodrizas) & 1 \\
\hline
\end{tabular}

\section{DISCUSIÓN}

A lo largo de este estudio, se han identificado dos unidades macro de análisis, por lo cual es imprescindible distinguir dos tipos de conclusiones, con la finalidad de ser congruentes con la línea que lleva este artículo.

\section{Conclusiones bibliográficas}

Sobre la base de los datos analizados a lo largo de este estudio, se identifica una tendencia significativa de aumento del número de estudios por año. Desde el 2004 se comienza a visualizar un incremento del número de estudios, que alcanza en 2009 un pico equivalente a diecisiete (17) artículos. En la gráfica de la Figura 1, si bien se observa un descenso del número de estudios en los últimos cinco años (2010-2014), ello puede atribuirse al hecho de que muchos de los estudios que se han elaborado recientemente no han sido incluidos aún en las bases de datos.

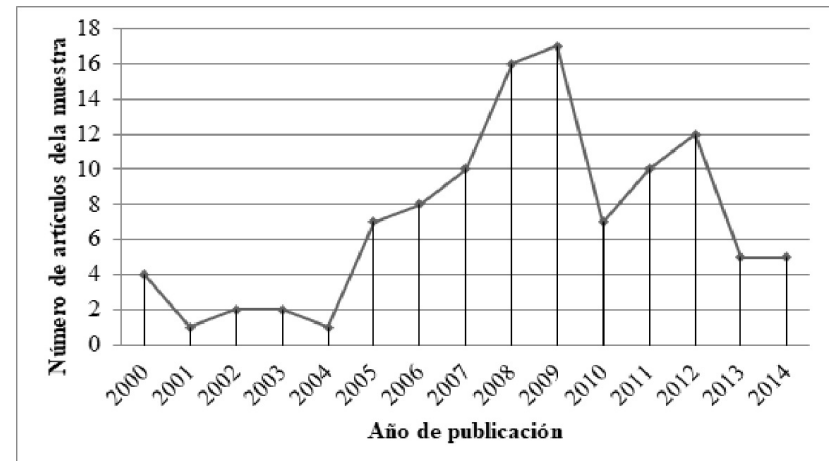

Figura 1. Número de artículos científicos de acuerdo al año de publicación (Intervalo 2000-2014). 
En lo que respecta a la distribución global, se observa que existe al menos un artículo publicado en PUBMED para cada continente (Ver Figura 2). A pesar de estar más concentrado en Europa y América, es válido afirmar que, primero, se está en presencia de un fenómeno que afecta a la población mundial y, segundo, que la muestra de 107 artículos, a pesar de ser significativa para la presente investigación, puede ser un porcentaje escaso para lo que representaría la totalidad de investigaciones empíricas sobre la automedicación.

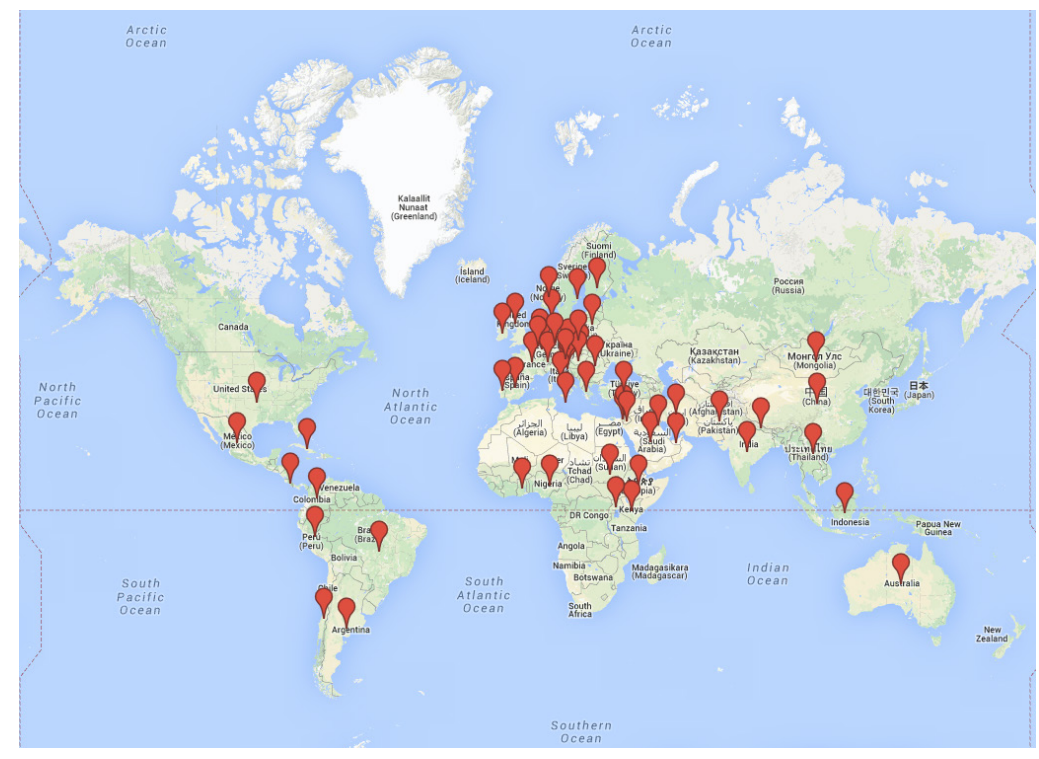

Figura 2. Distribución global de los 57 países en los que se ha realizado investigación de tipo empírico alrededor del fenómeno de automedicación.

Por otro lado, respecto a las revistas en las que se observan más publicaciones sobre automedicación, se identifican Pharmacy World and Science, que después del 2010 cambia su nombre a Internacional Journal of Clinical Pharmacy, y Family Practice, como cabeza de la lista. Sin embargo, no se observa en la muestra otra revista tan predominante como estas últimas.

Ahora bien, respecto a los datos arrojados acerca de los autores, se destacaron quince (15): Grigoryan, L., Bugerhorf,
J. G. M., Degener, J. E., Stalsby, C., Monnet, D. L., Scicluna, E. A., Birkin, J., HaaijerRuskamp, F. M., Bucaretchi, F., Stephan, C., Cordeiro, R., De Bolle, L., Mehuys, E., Remon, J-P., y Van Bortel, L. Es importante mencionar que algunos de estos autores se encuentran como productores de un mismo artículo, e incluso en dos publicaciones para años consecutivos, esto da indicios de grupos investigativos alrededor de temáticas específicas del gran fenómeno de automedicación. 


\section{Conclusiones por variables estudiadas en la muestra}

Inicialmente, la edad como variable revela que dentro de los grupos propuestos quien se lleva el primer lugar es el que tiene por etiqueta población general. La mayoría de los estudios se centran en población general, que en sí vendría definida como personas adultas, con edades de 18 años en adelante. No es difícil aceptar que las personas que se encuentran dentro de este grupo se les asignen como los más activos respecto a la práctica de automedicación. Esto, porque puede existir la tendencia de atribución, por su edad, de una capacidad de toma de decisiones más válida, estable y responsable. En este punto la existencia de estudios alrededor de la infancia y adolescencia cobra relevancia y llama la atención, lo que genera signos de interrogación acerca de qué variables influyen en que la población infantil y adolescente, a pesar de representar un pequeño porcentaje en la muestra, sean significativos, teniendo en cuenta que reflejan individuos con una capacidad de toma de decisiones muy baja o casi nula, dominada por los que en este estudio se conoce como población general.

En cuanto a los cuadros médicos extraídos de las investigaciones, se evidencia que algunos de estos constituyen condiciones de bajo riesgo, como resfriados, gripes, dolor de garganta y tos; no obstante, algunas otras condiciones son de mucho más cuidado, como las úlceras, problemas ginecológicos, enfermedades respiratorias e infecciones de transmisión sexual. Es importante destacar que se identifican, dentro de la lista, ítems que serían mejor clasificados como síntomas y no como enfermedades en sí. Ello podría interpretarse o darse a entender como todo esfuerzo que realiza la población, por medio de la automedicación, alrededor de sintomatología, para que no pase a una fase de desarrollo más avanzada y termine convirtiéndose en una enfermedad como tal. Por su parte, el comportamiento de automedicación de la población respecto a los ítems que representan enfermedades estructuradas se podría atribuir a la tendencia de esta hacia la promoción de la salud, prevención de la enfermedad, y estaría definida dentro de lo que se define como autocuidado.

Ahora bien, sobre los medicamentos identificados en la muestra se puede observar, a simple vista, que la lista abarca medicinas tanto tradicionales como alternativas, occidentales como orientales. Es decir que se reafirma el hecho de que la automedicación se trata de un tema transcultural. Los medicamentos que encabezan la lista son los antibióticos. Este tipo de medicamento suele ser uno de los más comunes en su uso y adquisición por parte de quienes se automedican para tratar enfermedades en distintos niveles y de diferente índole. El hecho de que sean los antibióticos la cabeza de la lista es preocupante, considerando que a pesar de tener efectos positivos en el organismo, como su efectividad al eliminar virus o simplemente aquello que genera molestia dentro del cuerpo, su uso indiscriminado dan lugar a reacciones internas en las que los mismos virus y bacterias generan resistencia y bloquean 
los efectos del antibiótico. Y, por tanto, ocasionan mayores problemáticas para el organismo. Es válido aclarar que el uso indiscriminado de los antibióticos puede hallar su origen en cogniciones erróneas, tales como que si un par de veces el antibiótico ha sido efectivo y ha funcionado para eliminar aquello que genera malestar, multiplicar su dosis o simplemente multiplicar las veces que se consume generará que lo que se considera enfermedad se elimine y, por tanto, se genere paralelamente una sensación de bienestar, sinónimo de salud.

Respecto a la variable de la ocupación de los sujetos de investigación, se observa que, en su mayoría, la población objeto pertenece al área de la salud. Dentro del sector de la salud se está generando la práctica en sí, este hecho puede deberse al fácil acceso a la variedad de medicamentos, tratamientos y conocimientos alrededor de la salud. En este preciso momento, cuando es válido cuestionarse hasta qué punto la automedicación en individuos vinculados al área de la salud es aconsejable. Por otro lado, las personas adscritas al área de la salud que aún no adquieren un título profesional, o que no se conciben como médicos profesionales, han sido uno de los factores más influyentes en el origen de la actividad de automedicación en la población general. Esta cuestión es preocupante, debido a que no poseen de manera formal todos los conocimientos que la práctica médica, en especial en el área farmacológica, les ofrece para brindar un mejor servicio a la comunidad. Sin embargo, no toda la "culpa" se le puede atribuir a los profesionales en formación o similares, se puede también pensar desde el panorama cultural de la persona que se automedica, y es que las personas pueden llegar a sentir seguridad cuando el sujeto que les aconseja un medicamento lleva un nombre que se relaciona con el campo de la salud (farmaceuta, estudiante de medicina, auxiliar de farmacia).

En lo que respecta a los estados especiales, se puede relacionar la existencia de estudios en etapas: estudios en etapas: infantil y adolescencia, que se reportan en la variable edades, previamente. Anteriormente se afirmaba que los niños y/o adolescentes que ejercen la automedicación no lo hacen por sí mismos, sino que existen variables que promueven dicha práctica. Aquí aparece esta variable que en este estudio se nombra como "estados especiales", que podrían ser aquellas variables que generan que la población infantil y adolescente se encuentre incluida en el ejercicio de la automedicación. Las personas que ejercen roles de paternidad/maternidad, cuidadores y mujeres en embarazo, poseen un acercamiento hacia algunas condiciones médicas, graves o no, que se pueden estar presentando ahora en los más pequeños, por lo cual pueden estar tomando planes de acción basados en experiencias, consejos guiados por publicidad, entre otros, para aliviar el dolor de quienes aman: sus hijos.

En conclusión, es importante destacar, dentro del análisis realizado, que la creciente investigación revela el interés del medio científico en el tema de 
automedicación. La atención a esta temática gira en torno a la multiplicación de usuarios que ejercen esta actividad y a los efectos y consecuencias de alto riesgo a los que se expone el usuario, o a los que el usuario expone su salud. Es una práctica que rodea las mejores intenciones por preservar la salud, por conservar el bienestar de sí mismo, pero que, aun así, la ignorancia que la rodea puede ser causante de consecuencias más perjudiciales que un no tratamiento.

Por último, es sorprendente el hecho de que la automedicación no se encuentre vinculada a condiciones de carencia de acceso a la salud. Es decir, la mayoría de la población no se automedica por no tener acceso a servicios de salud; por el contrario, parecen tener los recursos suficientemente significativos como para acceder a estos. Lo anterior surge como indicio, al encontrar el hecho de que la mayoría de la población de la muestra también tiene acceso a la educación universitaria. Se parte de una relación directamente proporcional: si la mayoría de individuos estudiados tienen los recursos suficientes para acceder a la educación superior (que representa un alto costo), tendrán los recursos suficientes para disfrutar de un acceso justo al sistema de atención en salud.
Se puede afirmar, pues, que no se trata de un fenómeno que se vincule con problemas económicos, ni de recursos inmediatos de los individuos y familias.

\section{Recomendaciones para futuros estudios}

Con base en los datos bibliográficos y variables analizados, se señala que la automedicación es un tema ampliamente investigado por una cantidad significativa de personas alrededor del mundo. Es importante sugerir la exigencia de más profundidad a la hora de establecer estudios empíricos, ya que hasta el momento se han percibido como una serie de estudios aislados.

Asimismo, la evidente dispersión de las investigaciones, sumada a la tendencia ascendente del número de referencias a través de los años, justificaría la propuesta de la creación de una revista especializada en la temática de investigación sobre la automedicación. Finalmente, la generalidad del fenómeno de la automedicación, reflejada en la muestra extraída para la presente investigación, alude a la necesidad de implementar investigaciones más precisas y puede proponerse la necesidad de realizar un estudio multicéntrico de tipo comparativo. 


\section{ReFERENCIAS}

Abahussain, E., Matowe, L.K., y Nicholls, P. J. (2005). Self-reported medication use among adolescents in Kuwait. Medical Principles and Practice, 14(3), 161-164. doi: $10.1159 / 000084633$

Afolabi, A.O. (2008). Factors influencing the pattern of self-medication in an adult Nigerian population. Annals of African Medicine, 7(3), 120-127. doi: 10.4103/15963519.55666

Aguilar-Palacio, I., Carrera-Lasfuentes, P., Poblador-Plou, B., Prados-Torres, A., y Rabanaque-Hernández, M.J. (2014). Morbidity and drug consumption. Comparison of results between the National Health Survey and electronic medical records. Gaceta Sanitaria, 28(1), 41-47. doi: 10.1016/j.gaceta.2013.04.004

Alghanim, S. A. (2011). Self-medication practice among patients in a public health care system. Eastern Mediterranean Health Journal, 17(5), 409-416.

Aljinović-Vucić, V., Trkulja, V., y Lacković, Z. (2005). Content of home pharmacies and self-medication practices in households of pharmacy and medical students in Zagreb, Croatia: Findings in 2001 with a reference to 1977. Croatian Medical Journal, 46(1), 74-80.

Anyama, N., y Adome, R. O. (2003). Community pharmaceutical care: an 8-month critical review of two pharmacies in Kampala. African Health Sciences, 3(2), 87-93.

Awad, A.I., y Eltayeb, I. B. (2007). Self-medication practices with antibiotics and antimalarials among Sudanese undergraduate university students. The Annals of Pharmacotherapy, 41(7), 1249-1255. doi: 10.1345/aph.1K068

Awad, A.I., Eltayeb, I. B., y Capps, P. A. (2006). Self-medication practices in Khartoum State, Sudan. European Journal of Clinical Pharmacology, 62(4), 317-324. doi: $10.1007 / \mathrm{s} 00228-006-0107-1$

Balbuena, F. R., Aranda, A. B., y Figueras, A. (2009). Self-medication in older urban mexicans: an observational, descriptive, cross-sectional study. Drugs Aging, 26(1), 51-60.

Bang, S., Sontakke, S., y Thawani, V. (2011). Pre and post-interventional pattern of self medication in three common illnesses in staff of a tertiary hospital. Indian Journal of Pharmacology, 43(3), 275-277. doi: 10.4103/0253-7613.81517

Baos, V. (2000). Estrategias para reducir los riesgos de la automedicación. Información terapéutica del Sistema Nacional de Salud, 24(6), 147-152.

Barbero-González, A., Pastor-Sánchez, R., Del Arco-Ortiz de Zárate, J., Eyaralar-Riera, T., y Espejo-Guerrero, J. (2006). Demand for dispensing of medicines without medical prescription. Atención Primaria, 37(2), 78-87. doi: 10.1157/13084485 
Bergmann, J. F. (2003). Self-medication: from European regulatory directives to therapeutic strategy. Fundamental \& Clinical Pharmacology, 17, 275-280.

Bertoldi, A. D., Silveira, M. P., Menezes, A. M., Assunçao, M. C., Gonçalves, H., y Hallal, P. C. (2012). Tracking of medicine use and self-medication from infancy to adolescence: 1993 Pelotas (Brazil) birth cohort study. Journal of Adolescent Health, 51(6), S11-15. doi: 10.1016/j.jadohealth.2012.06.027

Bi, P., Tong, S., y Parton, K. A. (2000). Family self-medication and antibiotics abuse for children and juveniles in a Chinese city. Social Science \& Medicine, 50(10), 1445-1450.

Bond, C., y Hannaford, P. (2003). Issues Related to Monitoring the Safety of Over-TheCounter (OTC) Medicines. Drug Safety, 26(15), 1065-1074.

Bortolon, P. C., Medeiros, E. F., Naves, J. O., Karnikowski, M. G., y Nóbrega Ode, T. (2008). Análise do perfil de automedicação em mulheres idosas brasileiras. Ciência \& Saúde Coletiva, 13(4), 1219-1226. doi: 10.1590/S1413-81232008000400018

Botero, D. (2005). Si la naturaleza es sabia, el hombre no lo es. En: L. Muñoz de Rodríguez, A. L. López, y O. J. Gómez, Cuidado de la vida. Cátedra Manuel Ancízar (pp. 15-34). Bogotá: Universidad Nacional de Colombia.

Carrasco-Garrido, P., Jiménez-García, R., Barrera, V. H., y Gil de Miguel, A. (2008). Predictive factors of self-medicated drug use among the Spanish adult population. Pharmacoepidemiology and Drug Safety, 17(2), 193-199. doi: 10.1002/pds.1455

Carrera-Lasfuentes, P., Aguilar-Palacio, I., Clemente Roldán, E., Malo Fumanal, S., y Rabanaque Hernandez, M. J. (2013). Medicine consumption in the adult population: Influence of self-medication. Atención primaria, 45(10), 528-535. doi: 10.1016/j. aprim.2013.05.006

Carvalho, R. S., Kara-José, N., Temporini, E. R., Kara-Junior, N. y Noma-Campos, R. (2009). Self-medication: initial treatments used by patients seen in an ophthalmologic emergency room. Clinics, 64(8), 735-741. doi: 10.1590/S1807-59322009000800005

Chaves, R. G., Lamounier, J. A. y César, C. C. (2009). Automedicação em nutrizes e sua influência sobre a duração do aleitamento materno. Jornal de Pediatria, 85(2), 129-134.

Collins, D., Oakey, S. y Ramakrishnan, V. (2011). Perioperative Use of Herbal, Complementary, and Over the Counter Medicines in Plastic Surgery Patients. Open Access Journal of Plastic Surgery, 11, 244-253.

Corrêa da Silva, M. G., Soares, M. C. y Muccillo-Baisch, A. L. (2012). Self-medication in university students from the city of Rio Grande, Brazil. BMC Public Health, 12, 339. doi: 10.1186/1471-2458-12-339 
Czeresnia, D. (1999). El concepto de salud y la diferencia entre prevención y promoción. Cuadernos de Salud Pública, 15(4), 701-710.

De Bolle, L., Mehuys, E., Adriaens, E., Remon, J. P., Van Bortel, L. y Christiaens, T. (2008). Home medication cabinets and self-medication: a source of potential health threats? Annals of Pharmacotherapy, 42(4), 572-579. doi: 10.1345/aph.1K533

De Roux, G. I. (1994). La prevención de comportamientos de riesgo y la promoción de estilos de vida saludables en el desarrollo de la Salud. Educación Médica y Salud, 28(2), 223-233.

Díaz-Caycedo, N., Payán-Madriñan, M. A., y Pérez-Acosta, A. M. (2014). Aproximación psicológica al comportamiento de automedicación. Revista Costarricense de Psicología, 33(1), 17-29.

Dieringer, N. J., Kukkamma, L., Somes, G. W., y Shorr, R. I. (2011). Self-reported responsiveness to direct-to-consumer drug advertising and medication use: results of a national survey. BMC Health Services Research, 11(1), 232. doi: 10.1186/1472-6963$11-232$

Donkor, E. S., Tetteh-Quarcoo, P. B., Nartey, P. y Agyeman, I. O. (2012). Self-medication practices with antibiotics among tertiary level students in Accra, Ghana: a cross-sectional study. International Journal of Environmental Research and Public Health, 9(10), 3519-3529. doi: 10.3390/ ijerph9103519

Du, Y., y Knopf, H. (2009). Self-medication among children and adolescents in Germany: results of the National Health Survey for Children and Adolescents (KiGGS). British Journal of Clinical Pharmacology, 68(4), 599-608. doi: 10.1111/j.13652125.2009.03477.x

Escobar, M. P., Franco, Z. R., y Duque, J. A. (2011). El autocuidado: Un compromiso de la formación integral en educación superior. Hacia la Promoción de la Salud, 16(2), 132-146.

Ethical Principles of Psychologists and Code of Conduct. (2002). Section 8. Research and Publication. Recuperado el 4 de noviembre de 2014 del URL: http://www.apa. org/ethics/code/principles.pdf.

Fainzang, S. (2013). The other side of Medicalization: Self-Medicalization and Self-Medication. Culture, Medicine and Psychiatry 37, 488-504.

Figueiras, A., Caamaño, F., y Gestal-Otero, J. J. (2000). Sociodemographic factors related to self-medication in Spain. European Journal of Epidemiology, 16(1), 19-26.

Fuentes Albarrán, K., y Villa Zapata, L. (2008). Analysis and quantification of self-medication patterns of customers in community pharmacies in southern Chile. Pharmacy World \& Science, 30(6), 863-868. doi: 10.1007/s11096-008-9241-4 
Geissler, P.W., Nokes, K., Prince, R. J., Odhiambo, R. A., Aagaard-Hansen, J., y Ouma, J.H. (2000). Children and medicines: self-treatment of common illnesses among Luo schoolchildren in western Kenya. Social Science \& Medicine, 50(12), 1771-1783.

Glover, D. D., Amonkar, M., Rybeck, B. F. y Tracy, T.S. (2003). Prescription, over-thecounter, and herbal medicine use in a rural, obstetric population. American Journal of Obstetrics \& Gynecology, 188(4), 1039-1045.

Goh, L.Y., Vitry, A. I., Semple, S. J., Esterman, A., y Luszcz, M. A. (2009). Self-medication with over-the-counter drugs and complementary medications in South Australia's elderly population. BMC Complementary and Alternative Medicine, 9, 42. doi: $10.1186 / 1472-6882-9-42$

Goldsworthy, R. C., y Mayhorn, C. B. (2009). Prescription Medication Sharing Among Adolescents: Prevalence, Risks, and Outcomes. Journal of Adolescent Health, 45(6), 634-637. doi: http://dx.doi.org/10.1016/j.jadohealth.2009.06.002

Gómez L. M., Galar, M. M., Téllez, A. M., Carmona, F. A., y Amaya, A. (2009). Estudio de automedicación en una farmacia comunitaria de la ciudad de Toluca. Revista Mexicana de Ciencias Farmacéuticas. 5-11.

González de Cossío, M. (2008). Nuevas etiquetas de medicamentos para apoyar la automedicación en México. El caso de un analgésico pediátrico. Salud Pública de México, 50(4), S453-S462.

González-López, J., Rodríguez-Gázquez, M. de A., y Lomas-Campos, M. de los A. (2012). Automedicación en inmigrantes latinoamericanos adultos de Sevilla. Acta Paulista de Enfermagem. 75-81.

Grahame-Smith, D. G. (1975). Self-medication with mood-changing drugs. Journal of Medical Ethics, 1(3), 132-137.

Granda, E. (2008). Automedicación. ¿Qué medicamentos y otros productos toman los ciudadanos para el cuidado de su salud y por qué? Farmacia Profesional, 22(1), 8-14.

Grigoryan, L., Burgerhof, J. G., Degener, J. E., Deschepper, R., Lundborg, C. S., Monnet, D. L., Scicluna, E. A., Birkin, J. y Haaijer-Ruskamp, F. M. (2007). Attitudes, beliefs and knowledge concerning antibiotic use and self-medication: a comparative European study. Pharmacoepidemiology and Drug Safety, 16(11), 1234-1243. doi: 10.1002/pds. 1479

Grigoryan, L., Burgerhof, J. G., Degener, J. E., Deschepper, R., Lundborg, C. S., Monnet, D. L., Scicluna, E. A., Birkin, J., y Haaijer-Ruskamp, F.M. (2008). Determinants of self-medication with antibiotics in Europe: the impact of beliefs, country wealth and the healthcare system. Journal of Antimicrobial Chemotherapy, 61(5), 1172-1179. doi: 10.1093/jac/dkn054 
Grigoryan, L., Burgerhof, J. G., Haaijer-Ruskamp, F. M., Degener, J. E., Deschepper, R., Monnet, D. L., Di Matteo, A., Scicluna, E. A., Bara, A. C., Lundborg, C. S. y Birkin, J. (2007). Is self-medication with antibiotics in Europe driven by prescribed use? Journal of Antimicrobial Chemotherapy, 59(1), 152-156. doi: 10.1093/jac/dk1457

Grzywacz, J. G., Arcury, T. A., Bell, R. A., Lang, W., Suerken, C. K., Smith, S. L., y Quandt, S. A. (2006). Ethnic differences in elders' home remedy use: sociostructural explanations. American Journal of Health Behavior, 30(1), 39-50. doi: 10.5555/ ajhb.2006.30.1.39

Gül, H., Omurtag, G., Clark, P. M., Tozan, A. y Ozel, S. (2007). Nonprescription medication purchases and the role of pharmacists as healthcare workers in self-medication in Istanbul. Medical Science Monitor, 13(7), 9-14.

Harris, K.M. y Edlund, M.J. (2005). Self-medication of mental health problems: new evidence from a national survey. Health Services Research, 40(1), 117-134. doi: 10.1111/j.1475-6773.2005.00345.x

Hem, E., Stokke, G., Tyssen, R., Gronvold, N. T., Vaglum, P., y Ekeberg, O. (2005). Self-prescribing among young norwegian doctors: a nine-year follow-up study of a nationwide sample. BMC Medicine, 3(16). doi: 10.1186/1741-7015-3-16

Hughes, C. M., McElnay, J. C., y Fleming, G.F. (2001). Benefits and risks of self-medication. Drug Safety, 24(14), 1027-1037.

Indermitte, J., Reber, D., Beutler, M., Bruppacher, R., y Hersberger, K.E. (2007). Prevalence and patient awareness of selected potential drug interactions with self-medication. Journal of Clinical Pharmacy and Therapeutics, 32(2), 149-159. doi: 10.1111/j.1365-2710.2007.00809.x

James, D. y French, D. (2008). The development of the Self-Medicating Scale (SMS): a scale to measure people's beliefs about self-medication. Pharmacy World \& Science, 30(6), 794-800.

Jiménez, L. G. y Fernández, X. (2008). Caracterización del uso de medicamentos en personas adultas mayores, Costa Rica 2007. Revista Costarricense de Salud Pública, $17,47-55$.

Jiménez Rubio, D. y Hernández Quevedo, C. (2010). Differences in self-medication in the adult population in Spain according to country of origin. Gaceta Sanitaria, 24(2), 116.e111-118. doi: 10.1016/j.gaceta.2009.09.007

Kivelevitch, D. N., Tahhan, P. V., Bourren, P., Kogan, N.N., Gusis, S.E. y Rodríguez, E. A. (2012). Self-medication and adherence to treatment in psoriasis. International Journal of Dermatology, 51(4), 416-419. doi: 10.1111/j.1365-4632.2011.05037.x 
Klemenc-Ketis, Z., Hladnik, Z., y Kersnik, J. (2011). A cross sectional study of sex differences in self-medication practices among university students in Slovenia. Collegium Antropologicum, 35(2), 329- 334.

Larson, E.L., Dilone, J., Garcia, M., y Smolowitz, J. (2006). Factors which influence Latino community members to self-prescribe antibiotics. Nursing Research and Practice, 55(2), 94-102.

Lechuga-Besné, M. A., Riveros-Rosas, A. y Sánchez-Sosa, J. J. (2009). Risk perception of the self-prescribed use of Over the Couter (OTC) cold/flu medications. International Journal of Clinical Health \& Psychology, 9(1), 73-88.

Lokker, N., Sanders, L., Perrin, E. M., Kumar, D., Finkle, J., Franco, V., Choi, L., Johnston, P. E., y Rothman, R. L. (2009). Parental misinterpretations of over-thecounter pediatric cough and cold medication labels. Pediatrics, 123(6), 1464-1471. doi: $10.1542 /$ peds.2008-0854

Loyola Filho, A. I., Lima-Costa, M. F., y Uchôa, E. (2004). Bambuí Project: a qualitative approach to self-medication. Cadernos de Saúde Pública, 20(6), 1661-1669. doi: / S0102-311X2004000600025

López, J. J., Dennis, R. y Moscoso, S. M. (2009). Estudio sobre la automedicación en una localidad de Bogotá. Revista de Salud Pública, 11, 432-442.

Mainous, A. G., Diaz, V. A. y Carnemolla, M. (2008). Factors affecting Latino adults' use of antibiotics for self-medication. The Journal of the American Board of Family Medicine, 21(2), 128-134. doi: 10.3122/jabfm.2008.02.070149

Mainous, A. G., Diaz, V. A., y Carnemolla, M. (2009). A community intervention to decrease antibiotics used for self-medication among Latino adults. The Annals of Family Medicine, 7(6), 520-526. doi: 10.1370/afm.1061

Major, C. y Vincze, Z. (2010a). Consumer habits and interests regarding non-prescription medications in Hungary. Family Practice, 27(3), 333-338. doi: 10.1093/fampra/ cmp105

Major, C. y Vincze, Z. (2010b). Self-medication in Hungary, from the perspective of pharmacy workers. Acta Poloniae Pharmaceutica, 67(5), 547-554.

Marin, M. J., Cecílio, L. C., Perez, A. E., Santella, F., Silva, C. B., Gonçalves Filho, J. R., y Roceti, L. C. (2008). Caracterização do uso de medicamentos entre idosos de uma unidade do Programa Saúde da Família. Cadernos de Saúde Pública, 24(7), 1545-1555.

Marquez, G. E., Torres, V. E., Sanchez, V. M., Gramajo, A. L., Zelaya, N., Peña, F. Y., Juarez, C. P., y Luna, J. D. (2012). Self-medication in ophthalmology: a questionnaire-based study in an Argentinean population. Ophthalmic Epidemiology, 19(4), 236-241. doi: 10.3109/09286586.2012.689076 
Matuz, M., Benko, R., Doro, P., Hajdu, E., y Soos, G. (2007). Non-prescription antibiotic use in Hungary. Pharmacy World \& Science, 29(6), 695-698. doi: 10.1007/s11096007-9132-0

McNulty, C. A., Boyle, P., Nichols, T., Clappison, P. y Davey, P. (2007). Don't wear me out. The public's knowledge of and attitudes to antibiotic use. Journal of Antimicrobial Chemotherapy, 59(4), 727-738. doi: 10.1093/jac/dk1558

Mehuys, E., Paemeleire, K., Van Hees, T., Christiaens, T., Van Bortel, L. M., Van Tongelen, I., De Bolle, L., Remon, J. P., y Boussery, K. (2012). Self-medication of regular headache: a community pharmacy-based survey. European Journal of Neurology, 19(8), 1093-1099. doi: 10.1111/j.1468-1331.2012.03681.x

Mehuys, E., Van Bortel, L., De Bolle, L., Van Tongelen, I., Remon, J. P., y De Looze, D. (2009). Self-medication of upper gastrointestinal symptoms: a community pharmacy study. The Annals of Pharmacotherapy, 43(5), 890-898. doi: 10.1345/aph.1L647

Miní, E., Varas, R., Vicuña, Y., Lévano, M., Rojas, L., Medina, J., Butron, J., Aranda, R., y Gutierrez, E. L. (2012). Self-medication behavior among pregnant women user of the Instituto Nacional Materno Perinatal, Perú 2011. Revista Peruana de Medicina Experimental y Salud Pública, 29(2), 212-217.

Ministerio de la Protección Social, República de Colombia. (2006). Ley 1090 de 2006. Recuperado el 31 de octubre de 2014, del URL: http:/www.secretariasenado.gov.co/ senado/basedoc/ley_1090_2006.html

Mintzes, B., Barer, M. L., Kravitz, R. L., Kazanjian, A., Bassett, K., Lexchin, J., Evans, R. G., Pan, R., y Marion, S. A. (2006). Influence of direct to consumer pharmaceutical advertising and patients' requests on prescribing decisions: two site cross sectional survey. British Medical Journal, 324(7332), 278-279.

Mira, J. J., Navarro, I. M., Guilabert, M., y Aranaz, J. (2012). Frecuencia de errores de los pacientes con su medicación. Revista Panamericana de Salud Pública, 31(2), 95-101.

Montgomery, A. J., Bradley, C., Rochfort, A., y Panagopoulou, E. (2011). A review of self-medication in physicians and medical students. Occupational Medicine, 61(7), 490-497. doi: 10.1093/occmed/kqr098

Moraes, A. C., Delaporte, T. R., Molena-Fernandes, C. A., y Falcão, M. C. (2011). Factors associated with medicine use and self-medication are different in adolescents. Clinics, 66(7), 1149-1155.

Morrison, D., Wyke, S., Agur, K., Cameron, E. J., Docking, R. I., Mackenzie, A. M., McConnachie, A. M, Raghuvir, V., Thomson, N. C., y Mair, F.S. (2014). Digital asthma self-management interventions: a systematic review. Journal of Medical Internet Research, 16(2), e51. doi: 10.2196/jmir.2814 
Neafsey, P. J., M'lan, C. E., Ge, M., Walsh, S. J., Lin, C. A., y Anderson, E. (2011). Reducing Adverse Self-Medication Behaviors in Older Adults with Hypertension: Results of an e-health Clinical Efficacy Trial. Ageing International, 36(2), 159-191.

Nunes de Melo, M., Madureira, B., Nunes Ferreira, A. P., Mendes, Z., Miranda Ada, C. y Martins, A. P. (2006). Prevalence of self-medication in rural areas of Portugal. Pharmacy World \& Science, 28(1), 19-25. doi: 10.1007/s11096-005-2222-y

Olivier, P., Bertrand, L., Tubery, M., Lauque, D., Montastruc, J. L. y Lapeyre-Mestre, M. (2009). Hospitalizations because of adverse drug reactions in elderly patients admitted through the emergency department: a prospective survey. Drugs Aging, 26(6), 475-482. doi: 10.2165/00002512-200926060-00004

Orueta, R., Gómez-Calcerrada, R. M., y Sánchez, A. (2008). Automedicación. Actualización en Medicina de Familia, 34(3), 133-137.

Pagán, J. A., Ross, S., Yau, J. y Polsky, D. (2006). Self-medication and health insurance coverage in Mexico. Health Policy, 75(2), 170-177. doi: 10.1016/j.healthpol.2005.03.007

Papakosta, M., Zavras, D., y Niakas, D. (2014). Investigating factors of self-care orientation and self-medication use in a Greek rural area. Rural Remote Health, 14(2), 2349.

Payne, J., Neutel, I., Cho, R., y Desmeules, M. (2004). Factors associated with women's medication use. BMC Women's Health, 4(S29).

Pereira, C. M., Alves, V. F., Gasparetto, P. F., Carneiro, D. S., de Carvalho, D.G. y Valoz, F.E. (2012). Self-medication in health students from two Brazilian universities. RSBO Revista Sul-Brasileira de Odontologia, 9(4), 361-367.

Pereira, F. S., Bucaretchi, F., Stephan, C., y Cordeiro, R. (2007). Self-medication in children and adolescents. Journal of Pediatrics, 83(5), 453-458. doi: 10.2223/ JPED.1703

Phrasisombath, K., Thomsen, S., Sychareun, V. y Faxelid, E. (2012). Care seeking behaviour and barriers to accessing services for sexually transmitted infections among female sex workers in Laos: a cross-sectional study. BMC Health Services Research, 12, 37. doi: 10.1186/1472-6963-12-37

Porteous, T., Bond, C., Hannaford, P. y Sinclair, H. (2005). How and why are non-prescription analgesics used in Scotland? Family Practice, 22(1), 78-85. doi: 10.1093/ fampra/cmh719

Quirós Enríquez, M., Fernández Ruíz, D., Cuevas Pérez, O., Milián Vázquez, P., Barrios Romero, B. y Martínez Barroso, T. (2012). Automedicación en trabajadores de la Facultad de Ciencias Médicas de Cienfuegos. MediSur, 10(6), 495-500.

Riedemann, J. P., Illesca, M. y Droghetti, J. (2001). Self-medication among patients with musculoskeletal symptoms in Araucanía region. Revista Médica de Chile, 129(6), 647-652. 
Sáiz, P. G., Bozal, F. F., Fernández, F. G. y Sánchez, C. S. (2010). Estudio sobre Automedicación en Población Universitaria Española. Revista Clínica de Medicina de Familia, 3(2), 99-103.

Sanfélix, J., Palop, V., Pereiró, B., y Martínez, I. (2002). Influencia del sexo del paciente en la calidad de los medicamentos consumidos. Atención Primaria, 30(3), 163-170.

Sapkota, A. R., Coker, M. E., Rosenberg Goldstein, R. E., Atkinson, N. L., Sweet, S. J., Sopeju, P. O., Ojo, M. T., Otivhia, E. Ayepola, O. O., Olajuyigbe, O. O., Shireman, L., Pottinger, P. S. y Ojo, K. K. (2010). Self-medication with antibiotics for the treatment of menstrual symptoms in Southwest Nigeria: a cross-sectional study. BMC Public Health, 10, 610. doi: 10.1186/1471-2458-10-610

Sawair, F. A., Baqain, Z. H., Abu Karaky, A. y Abu Eid, R. (2009). Assessment of self-medication of antibiotics in a Jordanian population. Medical Principles and Practice, 18(1), 21-25. doi: 10.1159/000163041

Sawalha, A. F. (2008). A descriptive study of self-medication practices among Palestinian medical and nonmedical university students. Research in Social and Administrative Pharmacy, 4(2), 164-172. doi: 10.1016/j.sapharm.2007.04.004

Schmid, B., Bernal, R. y Silva, N. N. (2010). Self-medication in low-income adults in Southeastern Brazil. Revista Saúde Pública, 44(6), 1039-1045.

Schneider, M., Bouvier Gallacchi, M., Goehring, C., Künzi, B. y Bovier, P.A. (2007). Personal use of medical care and drugs among Swiss primary care physicians. Swiss Medical Weekly, 137(7-8), 121-126. doi: 2007/07/smw-11606

Selvaraj, K., Kumar, S. G. y Ramalingam, A. (2014). Prevalence of self-medication practices and its associated factors in Urban Puducherry, India. Perspectives in Clinical Research, 5(1), 32-36. doi: 10.4103/2229-3485.124569

Shankar, P. R., Partha, P., y Shenoy, N. (2002). Self-medication and non-doctor prescription practices in Pokhara valley, Western Nepal: a questionnaire-based study. BMC Family Practice, 3, 17

Sharifi, A., Sharifi, H., Karamouzian, M., Mokhtari, M., Esmaeili, H. H., Nejad, A. S. y Rahmatian, M. (2013). Topical ocular anesthetic abuse among Iranian welders: time for action. Middle East African Journal of Ophthalmology, 20(4), 336-340. doi: 10.4103/0974-9233.120023

Sharma, R., Kapoor, B., y Verma, U. (2006). Drug utilization pattern during pregnancy in North India. Indian Journal of Medical Sciences, 60(7), 277-287.

Shehnaz, S. I., Khan, N., Sreedharan, J., Issa, K. J. y Arifulla, M. (2013). Self-medication and related health complaints among expatriate high school students in the United Arab Emirates. International Journal of Pharmacy Practice, 11(4), 211-218. 
Shelley, B. M., Sussman, A. L., Williams, R. L., Segal, A. R. y Crabtree, B. F. (2009). 'They don't ask me so I don't tell them': patient-clinician communication about traditional, complementary, and alternative medicine. The Annals of Family Medicine, 7(2), 139-147. doi: 10.1370/afm.947

Sihvo, S., Ahonen, R., Mikander, H. y Hemminki, E. (2000). Self-medication with vaginal antifungal drugs: physicians' experiences and women's utilization patterns. Family Practice, 17(2), 145-149.

Siliquini, R., Ceruti, M., Lovato, E., Bert, F., Bruno, S., De Vito, E., Liquori, G., Manzoli, L., Messina, G., Minniti, D. y La Torre, G. (2011). Surfing the internet for health information: an italian survey on use and population choices. BMC Medical Informatics and Decision Making, 11, 21.

Silva, I. de J., De Oliveira, M. F., Da Silva, S. E., Polaro, S. H., Radünz, V., Dos Santos, E. K., y De Santana, M. E. (2009). Cuidado, autocuidado y cuidado de sí: Una comprensión paradigmática para el cuidado de enfermería. Revista da Escola de Enfermagem da USP, 43(3), 697-703.

Soriano Hernández, R., Reyes-Hernández, U., Reyes Hernández, D., Reyes Gómez, U., García-Galavíz, J. L., y Garzón Sánchez, E. (2009). Frecuencia de automedicación en niños con infección respiratoria aguda en un área rural. Revista de la Facultad de Medicina UNAM, 52(3), 110-113.

Souza, L. A., Da Silva, C. D., Ferraz, G. C., Sousa, F. A. y Pereira, L. V. (2011). The Prevalence and Characterization of Self-Medication for Obtaining Pain Relief among Undergraduate Nursing Students. Revista Latino-Americana de Enfermagem (RLAE), 19(2), 245-251.

Suleman, S., Ketsela, A. y Mekonnen, Z. (2009). Assessment of self-medication practices in Assendabo town, Jimma zone, southwestern Ethiopia. Research in Social and Administrative Pharmacy, 5(1), 76-81. doi: 10.1016/j.sapharm.2008.04.002

Tawfik, K.A. y Jabeen, A. (2013). Pharmaceuticals safety practices-a comparative pilot study. International Journal of Health Sciences, 7(3), 317-324.

Tobón Marulanda, F. Á. (2002). Estudio sobre automedicación en la Universidad de Antioquia, Medellín, Colombia. Iatreia, 15(4), 242-247.

Tobón, O. (2003). El autocuidado una habilidad para vivir. Hacia la Promoción de la Salud, 8. Recuperado el 23 de agosto de 2014 de la URL: http://promocionsalud.ucaldas. edu.co/downloads/Revista\%208_5.pdf.

Togoobaatar, G., Ikeda, N., Ali, M., Sonomjamts, M., Dashdemberel, S., Mori, R. y Shibuya, K. (2010). Survey of non-prescribed use of antibiotics for children in an urban 
community in Mongolia. Bulletin of the World Health Organization, 88(12), 930-936. doi: 10.2471/BLT.10.079004

Tong, K. L., y Lien, C. Y. (1995). Do pharmaceutical representatives misuse their drug samples? Canadian Family Physician, 41, 1363-1366.

Tourinho, F. S., Bucaretchi, F., Stephan, C. y Cordeiro, R. (2008). Home medicine chests and their relationship with self-medication in children and adolescents. Journal of Pediatrics, 84(5), 416-422. doi:10.2223/JPED.1831

Ullah, H., Khan, S. A., Ali, S., Karim, S., Baseer, A., Chohan, O., Hassan, S. M., Khan, K. M. y Murtaza, G. (2013). Evaluation of self-medication amongst university students in Abbottabad, Pakistan; prevalence, attitude and causes. Acta Poloniae Pharmaceutica, 70(5), 919-922.

Uribe, T. M. (1999). El autocuidado y su papel en la promoción de la salud. Recuperado el 23 de agosto de 2014 del URL: http://www.bvsde.paho.org/bvsacd/cd26/ fulltexts/0467.pdf.

Vacas Rodilla, E., Castellà Dagà, I., Sánchez Giralt, M., Pujol Algué, A., Pallarés Comalada, M. C. y Balagué Corbera, M. (2009). Self-medication and the elderly. The reality of the home medicine cabinet. Atención Primaria, 41(5), 269-274. doi: 10.1016/j.aprim.2008.09.018

Verbrugge, L. M. (1985). Gender and health: an update on hypotheses and evidence. Journal of Health and Social Behavior, 26(3), 156-182.

Vitor, R. S., Lopes, C. P., Menezes, H. S. y Kerkhoff, C. E. (2008). Padrão de consumo de medicamentos sem prescrição médica na cidade de Porto Alegre, RS. Ciência \& Saúde Coletiva, 13, 737-743.

Vivancos, R., Schelenz, S. y Loke, Y. K. (2007). Internet treatment of sexually transmitted infections. A public health hazard? BMC Public Health, 7, 333. doi: 10.1186/14712458-7-333

Volpato, D. E., de Souza, B. V., Dalla Rosa, L. G., Melo, L. H., Daudt, C. A. y Deboni, L. (2005). Use of antibiotics without medical prescription. Brazilian Journal of Infectious Diseases, 9(4), 288-291.

Väänänen, M. H., Pietilä, K. y Airaksinen, M. (2006). Self-medication with antibiotics. Does it really happen in Europe? Health Policy, 77(2), 166-171. doi: 10.1016/j.healthpol.2005.07.001

Vega, O. M. y González, D. S. (2007). Teoría del déficit de autocuidado: interpretación desde los elementos conceptuales. Ciencia y Cuidado, 4(4), 28-35. 
Wazaify, M., Shields, E., Hughes, C. M. y McElnay, J. C. (2005). Societal perspectives on over-the-counter (OTC) medicines. Family Practice, 22(2), 170-176. doi: 10.1093/ fampra/cmh723

Widayati, A., Suryawati, S., de Crespigny, C. y Hiller, J. E. (2011). Self-medication with antibiotics in Yogyakarta City Indonesia: a cross sectional population-based survey. BMC Research Notes, 4, 491. doi: 10.1186/1756-0500-4-491

Wilbur, K., Al Tawengi, K. y Remoden, E. (2014). Diabetes patient management by pharmacists during Ramadan. BMC Health Services Research, 14(1), 117. doi: 10.1186/1472-6963-14-117

You, J. H., Wong, F. Y., Chan, F. W., Wong, E. L. y Yeoh, E. K. (2011). Public perception on the role of community pharmacists in self-medication and self-care in Hong Kong. BMC Clinical Pharmacology, 11, 19. doi: 10.1186/1472-6904-11-19

Yousef, A. M., Al-Bakri, A. G., Bustanji, Y. y Wazaify, M. (2008). Self-medication patterns in Amman, Jordan. Pharmacy World \& Science, 30(1), 24-30. doi: 10.1007/ s11096-007-9135-x

Zargarzadeh, A. H., Minaeiyan, M. y Torabi, A. (2008). Prescription and nonprescription drug use in isfahan, Iran: An observational, cross-sectional study. Current Therapeutic Research, 69(1), 76-87. doi: http://dx.doi.org/10.1016/j.curtheres.2008.02.002 


\section{Anexos}

Anexo A. Revistas más frecuentes en las que fueron publicados los artículos

\begin{tabular}{|c|c|}
\hline Nombre de la revista & $\begin{array}{c}\mathrm{N}^{\circ} \text { de artículos de la muestra adscritos } \\
\text { a esa revista }\end{array}$ \\
\hline Pharmacy World \& Science & 5 \\
\hline Family Practice & 4 \\
\hline Atención Primaria & 3 \\
\hline Annals of Pharmacotherapy & 3 \\
\hline Journal of Antimicrobial Chemotherapy & 3 \\
\hline Jornal de Pediatria (Rio J.) & 3 \\
\hline BMC Public Health & 3 \\
\hline BMC Health Services Research & 3 \\
\hline Social Science \& Medicine & 2 \\
\hline Cadernos de Saúde Pública & 2 \\
\hline Medical Principles and Practice & 2 \\
\hline Health Policy & 2 \\
\hline Indian Journal of Medical Sciences & 2 \\
\hline Pharmacoepidemiology and Drug Safety & 2 \\
\hline Ciência \& Saúde Coletiva & 2 \\
\hline $\begin{array}{l}\text { Research in Social and Administrative } \\
\text { Pharmacy }\end{array}$ & 2 \\
\hline Drugs \& Aging & 2 \\
\hline Clinics (Sao Paulo) & 2 \\
\hline Journal of Adolescent Health & 2 \\
\hline The Annals of Family Medicine & 2 \\
\hline Gaceta Sanitaria & 2 \\
\hline Acta Poloniae Pharmaceutica & 2 \\
\hline
\end{tabular}


Anexo B. Autores más frecuentes de los artículos de la muestra

\begin{tabular}{|c|c|}
\hline Nombre del autor & Número de veces que aparece en la muestra \\
\hline Grigoryan, L. & 3 \\
\hline Bugerhorf, J. G. M. & 3 \\
\hline Degener, J. E. & 3 \\
\hline Stalsby, C. & 3 \\
\hline Monnet, D.L. & 3 \\
\hline Scicluna, E.A. & 3 \\
\hline Birkin, J. & 3 \\
\hline Haaijer-Ruskamp, F. M. & 3 \\
\hline Bucaretchi, F. & 3 \\
\hline Stephan, C. & 3 \\
\hline Cordeiro, R. & 3 \\
\hline De Bolle, L. & 3 \\
\hline Mehuys, E. & 3 \\
\hline Remon, J-P. & 3 \\
\hline Van Bortel, L. & 3 \\
\hline Figueiras, A. & 2 \\
\hline Wazaify, M. & 2 \\
\hline Awad, A.I. & 2 \\
\hline Eltayeb, I. B. & 2 \\
\hline Deschepper, R. & 2 \\
\hline Pereira, F. S. & 2 \\
\hline Mainous, A. G. & 2 \\
\hline Díaz, V.A. & 2 \\
\hline Carnemolla, M. & 2 \\
\hline Neafsey, P. J. & 2 \\
\hline Anderson, E. & 2 \\
\hline Lin, C.A. & 2 \\
\hline M'lan, C. E. & 2 \\
\hline Walsh, S. & 2 \\
\hline
\end{tabular}


(continuación)

Van Tongelen, I. 2

Major, C. 2

Vincze, $\mathrm{Z}$. 2

Carrera-Lasfuentes, P. 2

Anexo C. Tipos de medicamentos evaluados como variable en la muestra

Tipo de medicamento

Antibióticos

Analgésicos

Vitaminas

Antiinflamatorios no esteroideos

Medicamentos para enfermedades gastrointestinales

Medicamentos herbarios Número de veces que aparece en la muestra

Tranquilizantes

Antialérgicos

Medicamentos para enfermedades respiratorias

Antiparásitos

Medicamentos para el resfriado

Anticonceptivos

Dermatológicos

Histaminas sistémicas

Antidepresivos

Remedios caseros

Laxantes

Antidiarreicos

Antianémicos

Minerales

Medicamentos homeopáticos

Antihipertensivos

Opiáceos

\section{8}

23

18

17

12

12

11

10

9

9

9

8

8

7

7

7

7

6

5

5

5

4

4 
(continuación)

Antihistamínicos

$\begin{array}{ll}\text { Medicamentos para enfermedades cardiovasculares } & 3\end{array}$

Antiespasmódicos 3

Medicamentos hormonales 3

Relajantes musculares 3

Antitrombolíticos 3

Antiácidos 3

$\begin{array}{ll}\text { Antimaláricos } & 2\end{array}$

$\begin{array}{ll}\text { Antibacterianos para uso sistémico } & 2\end{array}$

Medicamentos para alivio del dolor y la fiebre 2

Insulina 2

Benzodiacepinas 2

Diuréticos $\quad 2$

Hipoglicemiantes 2

Productos medicados para el cuidado de los ojos 2

Psicofármacos 2

Antimicrobiales $\quad 2$

Remedios para la tos $\quad 2$

Productos para el dolor de garganta 2

Antifúngico vaginal 1

Ansiolíticos 1

Broncodilatadores 1

Productos medicados para el cuidado de los pies 1

Productos medicados para el cuidado de los oídos 1

Medicamentos para las hemorroides 1

Tónicos 1

Antisépticos y desinfectantes 1

Antirreumáticos 1

Antidislipidémicos 1

Inmunomoduladores 1

$\begin{array}{ll}\text { Linimento } & 1\end{array}$ 
(continuación)

Antimicóticos 1

Medicina alternativa 1

Corticoesteroides 1

Vasoconstrictores 1

Anfetaminas 1

Antiulcerosos 1

Medicina alopática 1

Medicina ayurveda 1

Medicina umami 1

Suplementos nutricionales 1

Probióticos 1

Productos de combinación 1

Preparaciones nasales 1 